\title{
Bifurcation for some quasilinear operators
}

\author{
David Arcoya \\ Departamento de Análisis Matemático, Facultad de Ciencias, \\ C/Ochoa, 18071 Granada, Spain (darcoya@ugr.es)
}

\section{José Carmona}

Departamento de Álgebra y Análisis Matemático, Facultad de Ciencias, Cañada de San Urbano, Almeria, Spain (jcarmona@ual.es)

\section{Benedetta Pellacci}

Scuola Normale Superiore, Piazza Dei Cavalieri 7, 56126 Pisa, Italy (pellacci@cibs.sns.it)

(MS received 20 December 1999; accepted 4 October 2000)

This paper deals with existence, uniqueness and multiplicity results of positive solutions for the quasilinear elliptic boundary-value problem

$$
\begin{aligned}
-\operatorname{div}(A(x, u) \nabla u) & =f(\lambda, x, u) & & \text { in } \Omega, \\
u & =0 & & \text { on } \partial \Omega,
\end{aligned}
$$

where $\Omega$ is a bounded open domain in $\mathbb{R}^{N}$ with smooth boundary. Under suitable assumptions on the matrix $A(x, s)$, and depending on the behaviour of the function $f$ near $u=0$ and near $u=+\infty$, we can use bifurcation theory in order to give a quite complete analysis on the set of positive solutions. We will generalize in different directions some of the results in the papers by Ambrosetti et al., Ambrosetti and Hess, and Artola and Boccardo.

\section{Introduction}

Let $\Omega \subset \mathbb{R}^{N}$ be a bounded open set with sufficiently smooth boundary $\partial \Omega$ and let $f: \mathbb{R} \times \Omega \times \mathbb{R}^{+} \rightarrow \mathbb{R}$ be a Carathéodory function (i.e. $f(\lambda, x, s)$ is measurable with respect to $x$ for all $(\lambda, s) \in \mathbb{R} \times \mathbb{R}^{+}$and continuous in $(\lambda, s)$ for almost every $x \in \Omega)$, such that $f(\lambda, x, 0) \geqslant 0$ a.e. $x \in \Omega$ for every $\lambda \in \mathbb{R}^{+}$and satisfying, for some $r>\frac{1}{2} N$, that

for every bounded subset $\Lambda$ in $\mathbb{R}$ and for every $s_{0}>0$, there exists a positive function $C(x)$ belonging to $L^{r}(\Omega)$ such that

$$
|f(\lambda, x, s)| \leqslant C(x) \quad \text { a.e. } x \in \Omega \quad \forall \lambda \in \Lambda \quad \forall s \in\left[0, s_{0}\right] .
$$

We consider a symmetric matrix $A(x, s):=\left(a_{i j}(x, s)\right), i, j=1, \ldots, N$, with Carathéodory coefficients $a_{i j}: \Omega \times \mathbb{R}^{+} \rightarrow \mathbb{R}$ such that there exist positive constants $\alpha$ and $\beta$ satisfying, for every $(s, \xi) \in \mathbb{R}^{+} \times \mathbb{R}^{N}$ and a.e. $x \in \Omega$,

$$
\begin{gathered}
|A(x, s)| \leqslant \beta, \\
A(x, s) \xi \cdot \xi \geqslant \alpha|\xi|^{2} .
\end{gathered}
$$


In this paper we study the existence of positive solutions of the quasilinear boundary-value problem,

$$
\left.\begin{array}{rlrl}
-\operatorname{div}(A(x, u) \nabla u) & =f(\lambda, x, u), & & x \in \Omega, \\
u & =0, & & x \in \partial \Omega .
\end{array}\right\}
$$

To establish the definition of solution, we consider the usual Sobolev space $H_{0}^{1}(\Omega)$ and the space $C_{0}(\bar{\Omega})$ of the continuous functions in $\bar{\Omega}$ which vanish on $\partial \Omega$. Thanks to $\left(A_{1}\right)$ and $\left(f_{1}\right)$, for a positive solution of this problem, we mean a non-negative non-zero function $u \in H_{0}^{1}(\Omega) \cap C_{0}(\bar{\Omega})$ satisfying

$$
\int_{\Omega} A(x, u) \nabla u \cdot \nabla v=\int_{\Omega} f(\lambda, x, u) v \quad \forall v \in H_{0}^{1}(\Omega) .
$$

Problem $\left(P_{\lambda}\right)$ has been extensively studied in the semilinear case, i.e. the case in which the matrix $A$ does not depend on $s$, by means of bifurcation, variational and sub-supersolutions methods according to the behaviour of the function $f$. If the matrix $A(x, s)$ depends both on $x$ and $s$, problem $\left(P_{\lambda}\right)$ does not have variational structure in general and it is no more possible to use directly variational tools. The sub-supersolution method is applied in [7] for general matrices $A$ and particular classes of nonlinearities $f$. Specifically, the concave function $f_{1}(\lambda, x, s)=\lambda s^{q}$, with $0<q<1$, and the concave-convex function $f_{2}(\lambda, x, s)=\lambda s^{q}+s^{p}$, with $0<q<1<p$, are considered. In addition to $\left(A_{1}\right),\left(A_{2}\right)$, the authors impose the existence of an Osgood function $\omega: \mathbb{R}^{+} \rightarrow \mathbb{R}$, that is,

$$
\omega \text { is not decreasing, } \quad \omega(0)=0, \quad \int_{0^{+}} \frac{\mathrm{d} s}{\omega(s)}=+\infty,
$$

such that

$$
|A(x, s)-A(x, t)| \leqslant \omega(|s-t|) \quad \forall s, t \in \mathbb{R}^{+} .
$$

This condition is essential to prove a comparison theorem that allows them to use the sub and supersolutions techniques. Their results concern the existence of at least one positive solution for every positive $\lambda$ in the case $f=f_{1}$; while, if $f=f_{2}$, they find a positive number $\lambda^{*}$ such that problem $\left(P_{\lambda}\right)$ has at least one positive solution for every $\lambda \in\left(0, \lambda^{*}\right)$. The question of the uniqueness of the positive solution for $f=f_{1}$, and of the multiplicity of solutions for $f=f_{2}$ and $p<(N+2) /(N-2)$, as in the semilinear case (see $[2,10]$ ), remained open.

It seems, at least to the best knowledge of the authors, that bifurcation theory has not been used to study $\left(P_{\lambda}\right)$. One of the main technical difficulties in applying this functional approach is based on the nonlinearity of the differential operator $-\operatorname{div}(A(x, u) \nabla u)$. Indeed, this operator is not even homogeneous. Remember that the homogeneity of the involved differential operator is essential in applying the bifurcation techniques either in the semilinear case, like in [1], or in its quasilinear extensions $[3,4]$ for homogeneous operators, like the $p$-Laplacian one $\Delta_{p} \equiv \operatorname{div}\left(|\nabla u|^{p-2} \nabla u\right)$. (See also the work [14] for a different approach, also using the homogeneity of the differential operator.) Here, a careful analysis, by choosing suitable test functions, allows us to show the change of index that is needed to apply bifurcation theory [20], overcoming the difficulty of the lack of homogeneity. 
In this way, we show how bifurcation theory can be used to improve the previous results by giving a more complete analysis of the set of solutions and allowing us to handle more general classes of nonlinearities. Thus, in addition to generalizing the result in [1] for quasilinear operators like $-\operatorname{div}(A(x, u) \nabla u)$ and asymptotically linear functions $f$, either at zero (theorem 4.4) or at infinity (theorem 3.4), or both (theorem 5.1), we improve [7]. Specifically, in the case $f=f_{1}$, we recover (theorem 5.5) the same existence result as the one in [7]. Here, the new contribution is to point out that this result is a consequence of the existence of a connected set in $\mathbb{R} \times C_{0}(\bar{\Omega})$ of pairs $(\lambda, u)$, with $u$ a positive solution of $\left(P_{\lambda}\right)$, emanating from $(0,0)$, whose projection on the $\lambda$-axis contains the interval $(0,+\infty)$. In addition, under suitable assumptions on the matrix $A(x, s)$, we also prove a uniqueness theorem (theorem 5.8), which extends to the case of quasilinear operators the result in [10]. If $f=f_{2}$ and $p<(N+2) /(N-2)(N>2)$, we improve the results in [7] by showing in theorem 5.12 that there exist $\bar{\lambda}, \Lambda^{*}$ with $0<\bar{\lambda} \leqslant \Lambda^{*}$ such that problem $\left(P_{\lambda}\right)$ has at least two positive solutions for every $0<\lambda<\bar{\lambda}$ and no positive solutions if $\lambda>\Lambda^{*}$. Indeed, we find $\Lambda^{*}$ as an a priori estimate on the parameter $\lambda$ and we prove the existence of a connected set $\Sigma_{0}$ of positive solutions emanating from $u=0$ at $\lambda=0$, which, in virtue of an extension (theorem 5.11) of the Gidas-Spruck a priori estimate [16] for the positive solutions, turns back at a certain $\lambda=\bar{\lambda}$ to meet again $\lambda=0$ (see figure 6). Moreover, for a particular class of matrices of the kind $A(x, s)=A(x) a(s)$, we also prove (theorem 5.16) that $\bar{\lambda}=\Lambda^{*}$, i.e. the projection of $\Sigma_{0}$ on the $\lambda$-axis contains $\left(0, \Lambda^{*}\right]$, the maximal interval of positive $\lambda$ for which there is at least a positive solution of $\left(P_{\lambda}\right)$. We point out that this bifurcation approach to reach the maximal interval of positive $\lambda$ as the projection of $\Sigma_{0}$ (and consequently the existence of a second solution for $\lambda \in\left(0, \Lambda^{*}\right)$ ) seems to be unknown up to now, even in the semilinear case. These results extend to the quasilinear framework some of the results obtained in [2] for semilinear equations, where sub and supersolution techniques are adopted to obtain the maximal interval and variational tools are used to prove the existence of the second solution. Similar questions are also studied for radial $p$-Laplacian operator in balls in [3] (see, in particular, remark 3.13 of [3], where the authors set as an open question, in their context, whether the continuum emanating from zero at $\lambda=0$ gives the maximal interval of $\lambda$ ). We want to note that, since we use bifurcation techniques, we can also treat more general classes of nonlinearities $f$. Indeed, our existence results only require suitable hypotheses on the behaviour of $f$ near the origin and near infinity.

The paper is organized as follows. Section 2 is devoted to showing that, under the basic hypotheses $\left(A_{1}\right)-\left(A_{3}\right)$, we can reformulate $\left(P_{\lambda}\right)$ as a fixed-point problem. In $\oint \S 3$ and 4 we prove some abstract results concerning global bifurcation, namely, in $\S 3$ we study under which conditions it is possible to get a global bifurcation from infinity, while in $\S 4$ we are concerned with bifurcation from the trivial solution. In $\S 5$ we apply these results, together with some a priori estimates, in order to give a global analysis of the set of solutions of problem $\left(P_{\lambda}\right)$, and to obtain existence, multiplicity and uniqueness results depending on the behaviour of $f$.

\subsection{Notation}

In the sequel we will use the following notation. 
(i) $L^{r}(\Omega), 1 \leqslant r<\infty$, is the standard Lebesgue space and its norm will be denoted by $\|u\|_{r}$.

(ii) $H^{1}(\Omega), H_{0}^{1}(\Omega)$ are the usual Sobolev spaces. We denote by $2^{*}$ the Sobolev exponent related to $H^{1}(\Omega)$. We recall that $2^{*}=2 N /(N-2)$ if $N>2$ and $2^{*}=\infty$ if $N \leqslant 2 . H^{-1}(\Omega)$ is the dual space of $H^{1}(\Omega)$.

(iii) $H_{0}^{1}(\Omega)$ is endowed with the norm $\|u\|=\|\nabla u\|_{2}$.

(iv) $E:=C_{0}(\bar{\Omega})$ is the space of the continuous functions in $\bar{\Omega}$ that vanish on $\partial \Omega$, endowed with the norm $\|u\|_{0}=\sup _{\Omega}|u|$. For $R>0$, consider the open ball of radius $R, B_{R}=\{u \in E /\|u\|<R\}$.

(v) $C_{0}^{1}(\bar{\Omega})$ is the subspace of the functions in $C_{0}(\bar{\Omega})$ that are of class $C^{1}$ in $\bar{\Omega}$. Let $P$ denote the cone in $C_{0}^{1}(\bar{\Omega})$ of the non-negative functions, i.e.

$$
P=\left\{u \in C_{0}^{1}(\bar{\Omega}): u(x) \geqslant 0 \forall x \in \Omega\right\},
$$

whose interior is given by

$$
\dot{P}=\left\{u \in C_{0}^{1}(\bar{\Omega}): u(x)>0 \forall x \in \Omega, \frac{\partial u}{\partial n}(x)<0 \forall x \in \partial \Omega\right\},
$$

with $(\partial u / \partial n)(x)$ denoting the outward normal derivative of $u$ in the point $x \in \partial \Omega$.

(vi) We denote by $u^{+}, u^{-}$the positive and the negative parts of a function $u$ and we recall that $u^{-} \equiv \min \{u, 0\}$.

(vii) By $I$ we denote both the identity matrix and the identity operator. By $[A(x, s)-A(x)] \leqslant 0$ (respectively, $[A(x, s)-A(x)] \geqslant 0)$ we mean that the quadratic form induced by the matrix $A(x, s)-A(x)$ is definite non-positive (respectively, non-negative).

(viii) For operators $\Phi: E \rightarrow E$ of identity-compact type, $\operatorname{deg}\left(\Phi, B_{R}, 0\right)$ denotes the Leray-Schauder degree of $\Phi$ on $B_{R}$ with respect to zero, and $\mathrm{i}\left(\Phi, u_{0}\right)$ is the index of the isolated solution $u_{0}$ of the equation $\Phi(v)=0, v \in E$.

(ix) We also denote by Proj : $\mathbb{R} \times E \rightarrow \mathbb{R}$ the projection of the product space $\mathbb{R} \times E$ onto $\mathbb{R}$.

\section{Preliminaries}

Let $A$ be a matrix function satisfying $\left(A_{1}\right),\left(A_{2}\right)$. We extend the coefficients $a_{i j}(x, s)$ to all $\Omega \times \mathbb{R}$ by taking $a_{i j}(x, s) \equiv a_{i j}(x, 0)$ for every $x \in \Omega$ and $s<0$. Note that hypotheses $\left(A_{1}\right)$ and $\left(A_{2}\right)$ mean that the nonlinear operator $Q: H_{0}^{1}(\Omega) \rightarrow H^{-1}(\Omega)$, defined by

$$
Q(u)=-\operatorname{div}(A(x, u) \nabla u), \quad u \in H_{0}^{1}(\Omega),
$$

is continuous (by $\left(A_{1}\right)$ ) and coercive (by $\left(A_{2}\right)$ ). Then, from the classical result due to Leray and Lions [19], we deduce the existence of a (weak) solution $u$ of the problem

$$
Q(u)=h, \quad u \in H_{0}^{1}(\Omega),
$$

for every $h \in H^{-1}(\Omega)$. To be more specific, we have the following result. 
LEMma 2.1. Assume that A satisfies conditions $\left(A_{1}\right),\left(A_{2}\right)$ and let $h$ be in $H^{-1}(\Omega)$. Then there exists a weak solution $u \in H_{0}^{1}(\Omega)$ of problem (2.1), i.e. satisfying

$$
\int_{\Omega} A(x, u) \nabla u \cdot \nabla v=\int_{\Omega} h v \quad \forall v \in H_{0}^{1}(\Omega) .
$$

Furthermore, if hypothesis $\left(A_{3}\right)$ holds, then this solution $u$ is unique.

Proof. As we have observed, the existence is deduced from [19]. On the other hand, the uniqueness is discussed in [6].

REMARK 2.2. As a consequence of the preceding lemma, assuming $\left(A_{1}\right)-\left(A_{3}\right)$, we can consider the inverse $K: H^{-1}(\Omega) \rightarrow H_{0}^{1}(\Omega)$ of the operator $Q$. In addition, observe that, again by conditions $\left(A_{1}\right)-\left(A_{3}\right)$, the operator $K$ is continuous between $H^{-1}(\Omega)$ and $H_{0}^{1}(\Omega)$ and compact in $L^{2}(\Omega)$. Indeed, we only have to show that every sequence $h_{n}$ weakly convergent in $L^{2}(\Omega)$ to $h$ possesses a subsequence $h_{n_{k}}$ such that $K\left(h_{n_{k}}\right)$ strongly converges in $H_{0}^{1}(\Omega)$ to $K(h)$. To prove it, and writing $u_{n}=K\left(h_{n}\right)$ and $u=K(h)$, we take $u_{n}$ as a test function to deduce from $\left(A_{2}\right)$ that

$$
\begin{aligned}
\alpha\left\|u_{n}\right\|^{2} & \leqslant \int_{\Omega} A\left(x, u_{n}\right) \nabla u_{n} \cdot \nabla u_{n} \\
& =\int_{\Omega} h_{n} u_{n} \\
& \leqslant\left\|h_{n}\right\|_{2}\left\|u_{n}\right\|,
\end{aligned}
$$

and thus $u_{n}$ is bounded in $H_{0}^{1}(\Omega)$. Passing to a subsequence, if necessary, we can assume that $u_{n}$ weakly converges in this space to some $v \in H_{0}^{1}(\Omega)$. Now, using the fact that $u_{n}$ solves (2.1) for $h=h_{n}$, we obtain from the Rellich theorem that $v$ solves (2.1) for $h=h$, i.e. by $\left(A_{3}\right), v=K(h)=u$. Taking $u_{n}-u$ as a test function in the equation satisfied by $u_{n}$, condition $\left(A_{2}\right)$ yields

$$
\begin{aligned}
\alpha\left\|u_{n}-u\right\|^{2} & \leqslant \int_{\Omega} A\left(x, u_{n}\right) \nabla\left(u_{n}-u\right) \cdot \nabla\left(u_{n}-u\right) \\
& =\int_{\Omega} h_{n}\left(u_{n}-u\right)-\int_{\Omega} A\left(x, u_{n}\right) \nabla u \cdot \nabla\left(u_{n}-u\right) .
\end{aligned}
$$

Observe that, by Lebesgue's theorem and $\left(A_{2}\right)$, the sequence $A\left(x, u_{n}\right) \nabla u$ strongly converges to $A(x, u) \nabla u$ in $L^{2}(\Omega)$. Consequently, the right-hand side in the previous inequality tends to zero and thus $u_{n}$ strongly converges to $u$ in $H_{0}^{1}(\Omega)$.

REMARK 2.3. Moreover, if $h$ belongs to $L^{r}(\Omega)(r>N / 2)$, then, by the De GiorgiStampacchia theorem (see [21, theorem 7.3] and [13, theorem II] or $[17$, theorem 8.29]), the solution is a $C^{0, \alpha}(\bar{\Omega})$-function (for some $0<\alpha<1$ ). Furthermore, if the coefficients $a_{i j}$ are in $C^{1, \gamma}\left(\bar{\Omega} \times \mathbb{R}^{+}\right), 0<\gamma<1$, then, using a regularity theorem due to Campanato (see theorem 8.1 in [18] and [11]), we have that every solution $u$ of $Q(u)=h$ belongs to $C_{0}^{2, \alpha}(\bar{\Omega})$ for every $h \in C^{0, \gamma}(\bar{\Omega})$.

Now, consider a Carathéodory function $f$ in $\mathbb{R} \times \Omega \times \mathbb{R}^{+}$satisfying hypothesis $\left(f_{1}\right)$ and such that $f(\lambda, x, 0) \geqslant 0$ for a.e. $x \in \Omega$ and for every $\lambda \in \mathbb{R}^{+}$. Similarly, we extend the function $f$ to $\mathbb{R} \times \Omega \times \mathbb{R}$ by taking $f(\lambda, x, s)=f(\lambda, x, 0)$ for every $\lambda \in \mathbb{R}$, 
$x \in \Omega$ and $s<0$. In this way, we prove, by the maximum principle, that positive solutions $u$ of problem $\left(P_{\lambda}\right)\left(\lambda \in \mathbb{R}^{+}\right)$are just the zeros $u$ of the operator $\Phi_{\lambda}$ defined in $E$ by

$$
\Phi_{\lambda}(u) \equiv u-K(f(\lambda, x, u)), \quad u \in E,
$$

where, by simplicity, we denote in the same way the function $f$ and the Nemystskii operator related to it. Indeed, we have the following lemma.

Lemma 2.4. Assume that $A$ satisfies conditions $\left(A_{1}\right)-\left(A_{3}\right)$ and that $f$ is a Carathéodory function in $\mathbb{R} \times \Omega \times \mathbb{R}^{+}$satisfying hypothesis $\left(f_{1}\right)$ and that $f(\lambda, x, 0) \geqslant 0$ for a.e. $x \in \Omega$ and for every $\lambda \in \mathbb{R}^{+}$. Let $u \in E$ be a non-zero function and $\lambda \geqslant 0$. Then the following hold.

(i) $u$ is a positive solution of $\left(P_{\lambda}\right)$ if and only if

$$
\Phi_{\lambda}(u)=u-K(f(\lambda, x, u))=0 .
$$

(ii) If $u$ is a positive solution of $\left(P_{\lambda}\right)$, and, in addition, $a_{i j}$ and $f$ given in $\left(f_{1}\right)$ satisfy the following conditions,

$$
\left.\begin{array}{c}
C^{1, \gamma}\left(\bar{\Omega} \times \mathbb{R}^{+}\right), \quad 0<\gamma<1, \\
f(\lambda, \cdot, \cdot) \text { is a } C^{1} \text {-function in } \bar{\Omega} \times \mathbb{R}^{+} \quad \text { for every } \lambda>0,
\end{array}\right\}
$$

then $u \in \dot{P}$.

REMARK 2.5. Notice that if $u \in E$ then, by $\left(f_{1}\right), f(\lambda, x, u) \in L^{r}(\Omega)\left(r>\frac{1}{2} N\right)$ and regularity theorems imply that $K(f(\lambda, x, u)) \in E$. Thus $\Phi_{\lambda}: E \rightarrow E$ is well defined.

Proof. Let us prove part (i). Note that if $\Phi_{\lambda}(u)=0$, then $u \in H_{0}^{1}(\Omega) \cap E$ and it satisfies

$$
\int_{\Omega} A(x, u) \nabla u \cdot \nabla v=\int_{\Omega} f(\lambda, x, u) v \quad \forall v \in H_{0}^{1}(\Omega) .
$$

We claim that $u \geqslant 0$. Indeed, since $f(\lambda, x, s)=f(\lambda, x, 0) \geqslant 0$ a.e. $x \in \Omega$ and $s \leqslant 0$, we obtain from $\left(A_{2}\right)$, by taking $v=u^{-}$as a test function, that

$$
\begin{aligned}
\alpha\left\|u^{-}\right\|^{2} & \leqslant \int_{\Omega} A(x, u) \nabla u^{-} \cdot \nabla u^{-} \\
& \leqslant \int_{\Omega} f(\lambda, x, u) u^{-} \\
& \leqslant 0
\end{aligned}
$$

which implies $u^{-} \equiv 0$.

With respect to (ii), if $u \geqslant 0, u \neq \equiv 0$, solves $\left(P_{\lambda}\right)$, observe that the regularity of $f$ in (2.2) implies the existence of $m>0$ such that $f(\lambda, x, s)+m s$ is increasing in $s \in\left[0,\|u\|_{0}\right]$. Then, for $h(x)=f(\lambda, x, u(x))+m u(x)$ and $B(x)=A(x, u(x))$, we have

$$
-\operatorname{div}(B(x) \nabla u)+m u=h,
$$

where $0 \leqslant h \in C^{0, \alpha}(\bar{\Omega})$ and the coefficient $B$ is of $C^{1}$ class. Hence, by the strong maximum principle, $u>0$ in $\Omega$ and $(\partial u / \partial n)<0$ on $\partial \Omega$. This means that $u$ is in the interior of the cone $P$. 
REMARK 2.6. We want to point out that the regularity required to $f$ in condition (2.2) can be relaxed in the rest of the paper. It is sufficient to assume that, for every $s_{0} \in \mathbb{R}^{+}$, there exists $m \in \mathbb{R}^{+}$such that the function $f(\lambda, x, s)+m s$ is non-decreasing for $s \in\left[0, s_{0}\right]$.

\section{Bifurcation from infinity}

In this section we study bifurcation from infinity of positive solutions. We will prove the existence of a global bifurcation from infinity if the matrix $A(x, s)$ satisfies, in addition to $\left(A_{1}\right)-\left(A_{3}\right)$, the following condition:

$$
\exists \lim _{s \rightarrow+\infty} A(x, s)=A(x,+\infty) \quad \text { a.e. in } \Omega .
$$

With respect to $f$, we assume, in addition to $\left(f_{1}\right)$, that it is asymptotically linear at infinity, i.e. there exists a positive function $f_{\infty}^{\prime} \in L^{r}(\Omega)\left(r>\frac{1}{2} N\right)$, such that

$$
\lim _{s \rightarrow+\infty} \frac{f(\lambda, x, s)}{s}=\lambda f_{\infty}^{\prime}(x)
$$

uniformly with respect to $x \in \Omega$ and for every $\lambda \in[0,+\infty)$.

Concerning the dependence on $\lambda$, we suppose that there exist positive functions $K_{1}(x), K_{2}(x) \in L^{r}(\Omega)$ such that, for every $\lambda, \bar{\lambda} \in[0,+\infty)$,

$$
|f(\lambda, x, s)-f(\bar{\lambda}, x, s)| \leqslant|\lambda-\bar{\lambda}|\left[K_{1}(x) s+K_{2}(x)\right],
$$

for a.e. $x \in \Omega$ and for every $s \in \mathbb{R}^{+}$.

Note that conditions $\left(f_{1}\right)-\left(f_{3}\right)$ imply that if $\lambda$ lies in a bounded set $\Lambda$, there exist positive functions $C_{1}(x), C_{2}(x)$ in $L^{r}(\Omega)$ such that

$$
|f(\lambda, x, s)| \leqslant C_{1}(x) s+C_{2}(x)
$$

a.e. $x \in \Omega$, for every $s \in \mathbb{R}^{+}$and $\lambda \in \mathbb{R}$.

Notice that the condition $\left(f_{\infty}^{\prime}\right)^{+} \not \equiv 0$ implies that the weighted eigenvalue problem (see [12])

$$
\begin{aligned}
-\operatorname{div}(A(x,+\infty) \nabla u) & =\lambda f_{\infty}^{\prime}(x) u & & \text { in } \Omega, \\
u & =0 & & \text { on } \partial \Omega
\end{aligned}
$$

possesses a first positive eigenvalue $\lambda_{1}\left(f_{\infty}^{\prime}\right)$. In this section, we denote by

$$
\lambda_{\infty}=\lambda_{1}\left(f_{\infty}^{\prime}\right)
$$

We also denote by $\psi$ the positive eigenfunction with $\|\psi\|=1$ associated to $\lambda_{\infty}$.

LEMma 3.1. Assume that $\left(A_{1}\right)-\left(A_{4}\right)$ and $\left(f_{1}\right)-\left(f_{3}\right)$ hold. Let $\Lambda \subseteq\left[0, \lambda_{\infty}\right)$ be a compact interval. Then there exists a number $R>0$ such that, for every $u$ in $E$ with $\|u\|_{0} \geqslant R$ and for every $\lambda \in \Lambda, t \in[0,1]$,

$$
u \neq K(t f(\lambda, x, u))
$$


Proof. We argue by contradiction and we suppose that there exist sequences $\lambda_{n} \in \Lambda$, $t_{n} \in[0,1]$ and $u_{n} \in E$ with $\left\|u_{n}\right\|_{0} \rightarrow \infty$, such that

$$
u_{n}=K\left(t_{n} f\left(\lambda_{n}, x, u_{n}\right)\right) \text {. }
$$

Since $\Lambda$ and $[0,1]$ are compact sets, we deduce that there exist $\lambda \in \Lambda$ and $t \in[0,1]$ such that (up to a subsequence) $\lambda_{n} \rightarrow \lambda$ and $t_{n} \rightarrow t$. By the Stampacchia and De Giorgi theorems (see remark 2.3), from the convergence of $\left\|u_{n}\right\|_{0}$ to infinity, it derives also that $\left\|u_{n}\right\| \rightarrow \infty$. Moreover, if we define the normalized sequence $z_{n}:=u_{n}\left\|u_{n}\right\|^{-1}$, we have that $z_{n}$ verifies the following equation:

$$
\int_{\Omega} A\left(x, u_{n}\right) \nabla z_{n} \cdot \nabla v=t_{n} \int_{\Omega} \frac{f\left(\lambda_{n}, x, u_{n}\right)}{\left\|u_{n}\right\|} v \quad \forall v \in H_{0}^{1}(\Omega) .
$$

Using $\left(A_{2}\right)$ and $f(\lambda, x, s)=f(\lambda, x, 0) \geqslant 0$, for $\lambda \in \Lambda \subset[0, \infty), s<0$, we get, from the maximum principle, that $z_{n} \geqslant 0$.

Since $z_{n}$ is uniformly bounded in $H_{0}^{1}(\Omega)$, we can deduce (up to a subsequence) the existence of a function $z$ belonging to $H_{0}^{1}(\Omega)$ such that

$$
\begin{aligned}
z_{n} \rightarrow z & & \text { (weakly) in } H_{0}^{1}(\Omega), \\
z_{n}(x) \rightarrow z(x) \geqslant 0 & & \text { a.e. in } \Omega .
\end{aligned}
$$

Taking $v=z_{n}-z$ as a test function in (3.3) and using hypothesis $\left(A_{2}\right)$, we get

$$
\begin{aligned}
\alpha\left\|z_{n}-z\right\|^{2} & \leqslant \int_{\Omega} A\left(x, u_{n}\right) \nabla\left(z_{n}-z\right) \cdot \nabla\left(z_{n}-z\right) \\
& =t_{n} \int_{\Omega} \frac{f\left(\lambda_{n}, x, u_{n}\right)}{\left\|u_{n}\right\|}\left(z_{n}-z\right)-\int_{\Omega} A\left(x, u_{n}\right) \nabla z \cdot \nabla\left(z_{n}-z\right) .
\end{aligned}
$$

Thus we have, by (3.1),

$$
\alpha\left\|z_{n}-z\right\|^{2} \leqslant t_{n} \int_{\Omega}\left(C_{1}(x) z_{n}+\frac{C_{2}(x)}{\left\|u_{n}\right\|}\right)\left|z_{n}-z\right|-\int_{\Omega^{+}} A\left(x, u_{n}\right) \nabla z \cdot \nabla\left(z_{n}-z\right),
$$

where $\Omega^{+}:=\{x \in \Omega: z(x)>0\}$. Note that $u_{n}=\left\|u_{n}\right\| z_{n} \rightarrow+\infty$ almost everywhere in $\Omega^{+}$. Thus, thanks to hypotheses $\left(A_{1}\right)$ and $\left(A_{4}\right)$, we can apply the Lebesgue dominated convergence theorem to deduce that $A\left(x, u_{n}\right) \nabla z \rightarrow A(x, \infty) \nabla z$ strongly in $L^{2}\left(\Omega^{+}\right)$, and

$$
\int_{\Omega^{+}} A\left(x, u_{n}\right) \nabla z \cdot \nabla\left(z_{n}-z\right) \rightarrow 0 .
$$

Moreover, by the Rellich-Kondrachov compact embedding theorem and by the boundedness in $H_{0}^{1}(\Omega)$ of the sequence $z_{n}$, we also have that

$$
\lim _{n \rightarrow \infty} \int_{\Omega}\left(C_{1}(x) z_{n}+\frac{C_{2}(x)}{\left\|u_{n}\right\|}\right)\left|z_{n}-z\right|=0 .
$$


Therefore, from (3.4), we deduce that $z_{n} \rightarrow z$ strongly in $H_{0}^{1}(\Omega)$, so $z$ is not identically zero. Moreover, we get

$$
\begin{aligned}
\left|\int_{\Omega \backslash \Omega^{+}}\left[A\left(x, u_{n}\right)-A(x, \infty)\right] \nabla z_{n} \cdot \nabla v\right| & \leqslant 2 \beta \int_{\Omega \backslash \Omega^{+}}\left|\nabla z_{n}\right||\nabla v| \\
& \rightarrow 2 \beta \int_{\Omega \backslash \Omega^{+}}|\nabla z||\nabla v|=0
\end{aligned}
$$

and hence

$$
\lim _{n \rightarrow \infty} \int_{\Omega} A\left(x, u_{n}\right) \nabla z_{n} \cdot \nabla v=\int_{\Omega} A(x, \infty) \nabla z \cdot \nabla v \quad \forall v \in H_{0}^{1}(\Omega) .
$$

On the other hand, by $\left(f_{3}\right)$, we have

$$
\begin{aligned}
\left|\int_{\Omega} \frac{f\left(\lambda_{n}, x, u_{n}\right)}{\left\|u_{n}\right\|} v-\int_{\Omega} \frac{f\left(\lambda, x, u_{n}\right)}{\left\|u_{n}\right\|} v\right| & \leqslant \int_{\Omega} \frac{\left|f\left(\lambda_{n}, x, u_{n}\right)-f\left(\lambda, x, u_{n}\right)\right|}{\left\|u_{n}\right\|} v \\
& \leqslant\left|\lambda_{n}-\lambda\right| \int_{\Omega}\left[K_{1}(x) z_{n}+\frac{K_{2}(x)}{\left\|u_{n}\right\|}\right] v .
\end{aligned}
$$

Then, by the Sobolev embedding theorem and applying hypothesis $\left(f_{2}\right)$, we have

$$
\lim _{n \rightarrow \infty} \int_{\Omega} \frac{f\left(\lambda_{n}, x, u_{n}\right)}{\left\|u_{n}\right\|} v=\lambda \int_{\Omega} f_{\infty}^{\prime}(x) z v \quad \forall v \in H_{0}^{1}(\Omega) .
$$

Passing to the limit in (3.3), we obtain that the non-trivial and non-negative function $z$ verifies the following equation,

$$
-\operatorname{div}(A(x, \infty) \nabla z)=t \lambda f_{\infty}^{\prime}(x) z \quad \text { in } \Omega,
$$

which means that $t \lambda=\lambda_{\infty} \in \Lambda$ and $z=\psi$, i.e. a contradiction with the fact that $t \lambda<\lambda_{\infty}$

REMARK 3.2. The above lemma proves, in particular, that there are no bifurcation points from infinity for $\left(P_{\lambda}\right)$ at $\left(0, \lambda_{\infty}\right)$. However, we can prove even more. Specifically, there are no bifurcation points from infinity but, possibly, $\lambda_{\infty}$. Indeed, assume that $\lambda>0$ is a bifurcation point from infinity, i.e. there exists a sequence $\left(\lambda_{n}, u_{n}\right)$ such that $u_{n}$ is a positive solution of problem $\left(P_{\lambda}\right)$ for $\lambda=\lambda_{n},\left\|u_{n}\right\| \rightarrow \infty$ and $\lambda_{n} \rightarrow \lambda$. Following the same argument of lemma 3.1 (with $t=1$ ), we get that there exists $z \in H_{0}^{1}(\Omega)$ with $z>0,\|z\|=1$ and $z$ is a solution of the following problem:

$$
-\operatorname{div}(A(x, \infty) \nabla z)=\lambda f_{\infty}^{\prime}(x) z
$$

Then $z=\psi$ and $\lambda=\lambda_{\infty}$.

LEMMA 3.3. Let us assume that conditions $\left(A_{1}\right)-\left(A_{4}\right)$ and $\left(f_{1}\right)-\left(f_{3}\right)$ are satisfied and let $\phi$ be a positive function in $E \cap H_{0}^{1}(\Omega)$. If $\lambda>\lambda_{\infty}$, then there exists $R>0$ such that

$$
u-K(f(\lambda, x, u)+\tau \phi) \neq 0,
$$

for every $u$ in $E$ with $\|u\|_{0} \geqslant R$, for every $\tau \geqslant 0$. 
Proof. Let us argue by contradiction, and suppose that, for some sequence $u_{n}$ in $E$ with $\left\|u_{n}\right\|_{0} \rightarrow \infty$ and for a sequence of non-negative numbers $\tau_{n}$, we have

$$
-\operatorname{div}\left(A\left(x, u_{n}\right) \nabla u_{n}\right)=f\left(\lambda, x, u_{n}\right)+\tau_{n} \phi .
$$

Using $u_{n}^{-}$as a test function, we get

$$
\int_{\Omega} A\left(x, u_{n}\right) \nabla u_{n}^{-} \cdot \nabla u_{n}^{-}=\int_{\Omega} f\left(\lambda, x, u_{n}^{-}\right) u_{n}^{-}+\tau_{n} \int_{\Omega} u_{n}^{-} \phi .
$$

Since $\tau_{n} \geqslant 0$ and $\phi$ is positive, taking into account that $f(\lambda, x, s)=f(\lambda, x, 0) \geqslant 0$ for every $s \leqslant 0$, we deduce from condition $\left(A_{2}\right)$ that

$$
\alpha\left\|u_{n}^{-}\right\|^{2} \leqslant \int_{\Omega}\left(f(\lambda, x, 0)+\tau_{n} \phi\right) u_{n}^{-} \leqslant 0,
$$

and thus $u_{n} \geqslant 0$.

As in the proof of the previous lemma, by the Stampacchia and De Giorgi theorems, we have that $\left\|u_{n}\right\| \rightarrow \infty$, and if we define the normalized sequence $z_{n}:=u_{n}\left\|u_{n}\right\|^{-1}$, we can assume that $z_{n} \rightarrow z$ weakly in $H_{0}^{1}(\Omega)$ for some $z \in H_{0}^{1}(\Omega)$ and $z \geqslant 0$. Taking $\phi /\left\|u_{n}\right\|$ as a test function in (3.5) and using conditions $\left(A_{1}\right)$ and (3.1), we obtain that there exists a positive constant $C$ such that

$$
\frac{\tau_{n}}{\left\|u_{n}\right\|} \int_{\Omega} \phi^{2} \leqslant \int_{\Omega}\left(C_{1}(x) z_{n}+\frac{C_{2}(x)}{\left\|u_{n}\right\|}\right) \phi+\beta\|\phi\|^{2} \leqslant C .
$$

Then, up to a subsequence, there exists $\tau^{*} \geqslant 0$ such that $\tau_{n} /\left\|u_{n}\right\| \rightarrow \tau^{*}$.

Moreover, by taking $\left(z_{n}-z\right) /\left\|u_{n}\right\|$ as a test function in (3.5), we get

$$
\int_{\Omega} A\left(x, u_{n}\right) \nabla z_{n} \cdot \nabla\left(z_{n}-z\right)=\int_{\Omega} \frac{f\left(\lambda, x, u_{n}\right)}{\left\|u_{n}\right\|}\left(z_{n}-z\right)+\frac{\tau_{n}}{\left\|u_{n}\right\|} \int_{\Omega} \phi\left(z_{n}-z\right) .
$$

Subtracting $\int_{\Omega} A\left(x, u_{n}\right) \nabla z \cdot \nabla\left(z_{n}-z\right)$, we obtain from $\left(A_{2}\right)$ and (3.1) that

$$
\begin{aligned}
\alpha\left\|z_{n}-z\right\|^{2} \leqslant & \int_{\Omega} A\left(x, u_{n}\right) \nabla\left(z_{n}-z\right) \cdot \nabla\left(z_{n}-z\right) \\
= & \int_{\Omega} \frac{f\left(\lambda, x, u_{n}\right)}{\left\|u_{n}\right\|}\left(z_{n}-z\right) \\
& \quad+\frac{\tau_{n}}{\left\|u_{n}\right\|} \int_{\Omega} \phi\left(z_{n}-z\right)-\int_{\Omega} A\left(x, u_{n}\right) \nabla z \cdot \nabla\left(z_{n}-z\right) \\
\leqslant & \int_{\Omega}\left(C_{1}(x) z_{n}+\frac{C_{2}(x)}{\left\|u_{n}\right\|}\right)\left|z_{n}-z\right| \\
& \quad+\frac{\tau_{n}}{\left\|u_{n}\right\|} \int_{\Omega} \phi\left(z_{n}-z\right)-\int_{\Omega} A\left(x, u_{n}\right) \nabla z \cdot \nabla\left(z_{n}-z\right) .
\end{aligned}
$$

By the Rellich-Kondrachov compact embedding theorem, we have

$$
\lim _{n \rightarrow \infty} \int_{\Omega}\left(C_{1}(x) z_{n}+\frac{C_{2}(x)}{\left\|u_{n}\right\|}\right)\left|z_{n}-z\right|=0, \quad \lim _{n \rightarrow \infty} \int_{\Omega} \phi\left(z_{n}-z\right)=0 .
$$


Conditions $\left(A_{1}\right)$ and $\left(A_{4}\right)$ imply that $A\left(x, u_{n}\right) \nabla z$ strongly converges in $L^{2}\left(\Omega^{+}\right)$to $A(x, \infty) \nabla z$, where $\Omega^{+}=\{x \in \Omega: z(x)>0\}$. Then we get

$$
\lim _{n \rightarrow \infty} \int_{\Omega} A\left(x, u_{n}\right) \nabla z \cdot \nabla\left(z_{n}-z\right)=\lim _{n \rightarrow \infty} \int_{\Omega^{+}} A\left(x, u_{n}\right) \nabla z \cdot \nabla\left(z_{n}-z\right)=0 .
$$

Hence (3.6) yields the strong convergence of $z_{n}$ to $z$ in $H_{0}^{1}(\Omega)$ and $z$ is not identically zero. Dividing (3.5) by $\left\|u_{n}\right\|$ and passing to the limit, we get that $z$ solves the following equation:

$$
-\operatorname{div}(A(x, \infty) \nabla z)=\lambda f_{\infty}^{\prime}(x) z+\tau^{*} \phi
$$

Using $\psi$ as a test function, we obtain, since $z \not \equiv 0$, that

$$
\lambda_{\infty} \int_{\Omega} f_{\infty}^{\prime}(x) z \psi \geqslant \lambda \int_{\Omega} f_{\infty}^{\prime}(x) z \psi,
$$

which is a contradiction.

Now, combining the two previous lemmas, we can derive sufficient conditions to have a global bifurcation from infinity at $\lambda=\lambda_{\infty}$ of positive solutions. In addition, following the ideas of [5], we can also determine if the bifurcation is to the left or to the right of $\lambda=\lambda_{\infty}$.

We will denote by $\Sigma$ the closure of the set

$$
\left\{(\lambda, u) \in \mathbb{R} \times E \text { such that } \Phi_{\lambda}(u)=0, u \neq 0\right\} .
$$

TheOREm 3.4. Assume that conditions $\left(A_{1}\right)-\left(A_{4}\right)$ and $\left(f_{1}\right)-\left(f_{3}\right)$ hold and that $f(\lambda, x, 0) \geqslant 0$ for a.e. $x \in \Omega$, and for every $\lambda \in \mathbb{R}_{0}^{+}$. Then $\lambda_{\infty}=\lambda_{1}\left(f_{\infty}^{\prime}\right)$ is a bifurcation point from infinity of positive solutions, and it is the only one in $\mathbb{R}_{0}^{+}$. Furthermore, there exists an unbounded component $\Sigma_{\infty} \subset \Sigma$ such that

$$
\tilde{\Sigma}_{\infty}=\left\{(\lambda, u) \text { with } u \neq 0,\left(\lambda, \frac{u}{\|u\|^{2}}\right) \in \Sigma_{\infty}\right\} \cup\left\{\left(\lambda_{\infty}, 0\right)\right\}
$$

is connected and, if $f(0, x, s) \equiv 0$ for every $x \in \Omega$ and $s \geqslant 0$, unbounded. In addition, if the coefficients of the matrix $A(x, \infty)$ are of class $C^{1}$ and $r>N(r$ given in $\left.\left(f_{1}\right)\right)$, the following conditions hold.

(i) If (2.2) holds and there exist $\varepsilon_{0}>0, \sigma \in(0,3-1 / r)$ and $\underline{C}(x) \in L^{r}(\Omega)$ such that, denoting

$$
\underline{\mu}(x) \equiv \liminf _{(\lambda, s) \rightarrow\left(\lambda_{\infty}, \infty\right)}\left[f(\lambda, x, s)-\lambda f_{\infty}^{\prime}(x) s\right] s^{\sigma-1},
$$

for a.e. in $\Omega$, and for all $s>0$ and $\lambda \in\left(\lambda_{\infty}-\varepsilon_{0}, \lambda_{\infty}+\varepsilon_{0}\right)$, we have

$$
\left.\begin{array}{rl}
{[A(x, s)-A(x, \infty)]} & \leqslant 0, \\
{\left[f(\lambda, x, s)-\lambda f_{\infty}^{\prime}(x) s\right] s^{\sigma-1}} & \geqslant \underline{C}(x), \\
\int_{\Omega} \underline{\mu}(x) \psi^{2-\sigma}(x) & >0
\end{array}\right\}
$$

then the bifurcation of positive solutions is subcritical (i.e. to the left of $\left.\lambda=\lambda_{\infty}\right)$. 
(ii) If (2.2) holds and there exist $\varepsilon_{1}>0, \sigma \in(0,3-1 / r)$ and $\bar{C}(x) \in L^{r}(\Omega)$ such that, denoting

$$
\bar{\mu}(x) \equiv \limsup _{(\lambda, s) \rightarrow\left(\lambda_{\infty}, \infty\right)}\left[f(\lambda, x, s)-\lambda f_{\infty}^{\prime}(x) s\right] s^{\sigma-1},
$$

for a.e. in $\Omega$, and for all $s>0$ and $\lambda \in\left(\lambda_{\infty}-\varepsilon_{1}, \lambda_{\infty}+\varepsilon_{1}\right)$, we have

$$
\begin{aligned}
{[A(x, s)-A(x, \infty)] } & \geqslant 0, \\
{\left[f(\lambda, x, s)-\lambda f_{\infty}^{\prime}(x) s\right] s^{\sigma-1} } & \leqslant \bar{C}(x), \\
\int_{\Omega} \bar{\mu}(x) \psi^{2-\sigma}(x) & <0,
\end{aligned}
$$

then the bifurcation of positive solutions is supercritical (i.e. to the right of $\left.\lambda_{\infty}\right)$.

REMARK 3.5. The fact that $\left(\lambda_{\infty}, 0\right) \in \tilde{\Sigma}_{\infty}$ means that $\Sigma_{\infty}$ emanates from $\infty$ at $\lambda_{\infty}$.

REMARK 3.6. Since $\psi \in \dot{P}$, if $r^{\prime}$ is the conjugate exponent of $r$, i.e. $r^{\prime}=r /(r-1)$, then

$$
\psi^{2-\sigma} \in L^{r^{\prime}}(\Omega) \Longleftrightarrow \sigma<3-\frac{1}{r} .
$$

Thus the range of values of the exponent $\sigma$ is strictly related to the integrability of the function $\psi^{2-\sigma}$. In this way, for $\sigma<3-1 / r$, if there exists

$$
\lim _{(\lambda, s) \rightarrow\left(\lambda_{\infty}, \infty\right)}\left[f(\lambda, x, s)-\lambda f_{\infty}^{\prime}(x) s\right] s^{\sigma-1}=\mu \in \mathbb{R}^{*}
$$

for a.e. $x \in \Omega$, then items (i) and (ii) imply that the side of the bifurcation from infinity is decided by the sign of $\mu$. The condition $\sigma<3-1 / r$ is essential to deduce the side of the bifurcation from hypotheses on the behaviour of $f$ (and $A$ ) at $s=+\infty$. Indeed, for the case $A(x, s)=I, r=\infty$, it is proved in [5] that if $\sigma \geqslant 3$, it is not possible to determine whether the bifurcation is supercritical (or subcritical) by just assuming the hypothesis on the behaviour of the nonlinearity for large $s$, as in the third condition of (3.7).

Proof. In order to study the bifurcation from infinity, we follow the standard pattern (see, for example, [1]) and we perform the change of variable $z:=\|u\|^{-2} u(u \neq 0)$. We thus consider the map $\Psi_{\lambda}: E \rightarrow E$ defined by

$$
\Psi_{\lambda}(z)= \begin{cases}z-\|z\|^{2} K\left(f\left(\lambda, x, z /\|z\|^{2}\right)\right), & z \neq 0 \\ 0, & z=0\end{cases}
$$

By remark 3.2 we have that $\lambda_{\infty}$ is the only possible bifurcation point from infinity. To prove that $\lambda_{\infty}$ is actually a bifurcation point from infinity, we show that the index of the trivial solution for the equation $\Psi_{\lambda}(u)=0$ changes when $\lambda$ crosses $\lambda=\lambda_{\infty}$. Indeed, if $0<\lambda<\lambda_{\infty}$, applying lemma 3.1 to the compact set $\Lambda=\{\lambda\}$, we obtain the existence of a positive number $R>0$ such that, for every $t \in[0,1]$ and for every $u$ in $E$ with $\|u\|_{0} \geqslant R$,

$$
u-K(t f(\lambda, x, u)) \neq 0 \text {. }
$$


Thus we obtain from the homotopy invariance of the Leray-Schauder degree that

$$
\operatorname{deg}\left(\Psi_{\lambda}, B_{\varepsilon}, 0\right)=\operatorname{deg}\left(I, B_{\varepsilon}, 0\right)=1 \quad \forall \varepsilon \in\left(0, R^{-1}\right],
$$

and consequently,

$$
\mathrm{i}\left(\Psi_{\lambda}, 0\right)=1 \quad \forall \lambda \in\left(0, \lambda_{\infty}\right) .
$$

On the other hand, if $\lambda>\lambda_{\infty}$, from lemma 3.3, we derive the existence of $R_{0}>0$ such that

$$
u \neq K(f(\lambda, x, u)+t \phi)
$$

for every $u \in E$ with $\|u\|_{0} \geqslant R_{0}$, and for every $t \in[0,1]$. Thus, if we define the homotopy $H$ in $[0,1] \times E$ by

$$
H(t, z)= \begin{cases}z-\|z\|^{2} K\left(f\left(\lambda, x, z /\|z\|^{2}\right)+t \phi\right), & z \neq 0 \\ 0, & z=0\end{cases}
$$

then this homotopy is admissible in $[0,1] \times B_{\varepsilon}$, for $\varepsilon \in\left(0, R_{0}^{-1}\right]$. Moreover, lemma 3.3 shows that $H(1, \cdot)$ has no zeros in $B_{\varepsilon}$. Hence we have

$$
\operatorname{deg}\left(\Psi_{\lambda}, B_{\varepsilon}, 0\right)=\operatorname{deg}\left(H(0, \cdot), B_{\varepsilon}, 0\right)=\operatorname{deg}\left(H(1, \cdot), B_{\varepsilon}, 0\right)=0 .
$$

Therefore, we have

$$
\mathrm{i}\left(\Psi_{\lambda}, 0\right)=0 \quad \forall \lambda>\lambda_{\infty}
$$

and the conclusion can be obtained following the proof of proposition 3.5 in [1].

As for the study of the side of the bifurcation, i.e. of items (i) and (ii), we prove only assertion (i); the other one can be proved in a similar way and it is left to the reader. Let us argue by contradiction and consider a sequence $\left(\lambda_{n}, u_{n}\right)$ of solutions of the equation $\Phi_{\lambda}(u)=0$, i.e. satisfying

$$
\int_{\Omega} A\left(x, u_{n}\right) \nabla u_{n} \cdot \nabla v=\int_{\Omega} f\left(\lambda_{n}, x, u_{n}\right) v \quad \forall v \in H_{0}^{1}(\Omega),
$$

with $\lambda_{n}$ converging to $\lambda_{\infty}, \lambda_{n} \geqslant \lambda_{\infty}$ and $\left\|u_{n}\right\|_{0} \rightarrow+\infty$. By (2.2), every solution $u_{n}$ belongs to the interior of the cone $P$ (see lemma $2.4(\mathrm{ii})$ ). Thus we can take $v=\psi^{2} / u_{n}$ as a test function in (3.8), and we get

$$
\int_{\Omega} A\left(x, u_{n}\right) \nabla u_{n} \cdot\left[2 \frac{\psi}{u_{n}} \nabla \psi-\left(\frac{\psi}{u_{n}}\right)^{2} \nabla u_{n}\right]=\int_{\Omega} f\left(\lambda_{n}, x, u_{n}\right) \frac{\psi^{2}}{u_{n}} .
$$

Hence, denoting

$$
g\left(\lambda_{n}, x, u_{n}\right):=\left[f\left(\lambda_{n}, x, u_{n}\right)-\lambda_{n} f_{\infty}^{\prime}(x) u_{n}\right] u_{n}^{\sigma-1},
$$

we yield

$$
\begin{aligned}
\int_{\Omega} g\left(\lambda_{n}, x, u_{n}\right) \frac{\psi^{2}}{u_{n}^{\sigma}}=\int_{\Omega} & {\left[A\left(x, u_{n}\right)-\frac{\lambda_{n}}{\lambda_{\infty}} A(x, \infty)\right] \nabla \psi \cdot \nabla \psi } \\
& \quad-\int_{\Omega} A\left(x, u_{n}\right)\left(\nabla \psi-\frac{\psi}{u_{n}} \nabla u_{n}\right) \cdot\left(\nabla \psi-\frac{\psi}{u_{n}} \nabla u_{n}\right) .
\end{aligned}
$$


By the fact that $\lambda_{n} \geqslant \lambda_{\infty}$, we get, applying conditions $\left(A_{2}\right)$ and $(3.7)$, that

$$
\begin{aligned}
0 \geqslant \int_{\Omega}\left[A\left(x, u_{n}\right)-\frac{\lambda_{n}}{\lambda_{\infty}}\right. & A(x, \infty)] \nabla \psi \cdot \nabla \psi \\
& \quad-\int_{\Omega} A\left(x, u_{n}\right)\left(\nabla \psi-\frac{\psi}{u_{n}} \nabla u_{n}\right) \cdot\left(\nabla \psi-\frac{\psi}{u_{n}} \nabla u_{n}\right) .
\end{aligned}
$$

In particular,

$$
0 \geqslant \liminf _{n \rightarrow \infty} \int_{\Omega} g\left(\lambda_{n}, x, u_{n}\right) \frac{\psi^{2}}{z_{n}^{\sigma}},
$$

where, as before, $z_{n}=u_{n}\left\|u_{n}\right\|^{-1}$. Using the fact that $z_{n}$ converges uniformly to $\psi$ and Fatou's lemma, we obtain, thanks to (3.7), that

$$
0 \geqslant \liminf _{n \rightarrow \infty} \int_{\Omega} g\left(\lambda_{n}, x, u_{n}\right) \frac{\psi^{2}}{z_{n}^{\sigma}} \geqslant \int_{\Omega} \underline{\mu}(x) \psi^{2-\sigma}>0,
$$

which is a contradiction.

\section{Bifurcation from the trivial solution}

We assume in this section that hypotheses $\left(A_{1}\right)-\left(A_{3}\right)$ and $\left(f_{1}\right)$ hold. In addition, we suppose that the nonlinearity $f$ satisfies $f(\lambda, x, 0)=0$, for a.e. $x \in \Omega$ and for every $\lambda \in \mathbb{R}$, and the following condition.

For every $\Lambda$ bounded set of $\mathbb{R}-\{0\}$ and $\lambda \in \Lambda$,

$$
\lim _{s \rightarrow 0^{+}} \frac{f(\lambda, x, s)}{s}=\lambda f_{+}^{\prime}(x, 0), \text { uniformly in }(\lambda, x) \in \Lambda \times \Omega,
$$

with either $0 \leqslant f_{+}^{\prime}(x, 0) \in L^{r}(\Omega), r>\frac{1}{2} N$, not identically zero or $f_{+}^{\prime}(x, 0)=+\infty$ a.e. $x \in \Omega$.

For the case of integrable weight $f_{+}^{\prime}(x, 0)$, we also impose a similar condition to $\left(f_{3}\right)$.

If $f_{+}^{\prime}(x, 0) \in L^{r}(\Omega)$, with $r>\frac{1}{2} N$, then the limit in $\left(f_{4}\right)$ is uniformly in every bounded set $\Lambda \subset \mathbb{R}$ and there exist a positive function $K(x) \in$ $L^{r}(\Omega)$ and $\varepsilon_{0}>0$ such that, for every $\lambda, \bar{\lambda} \in \mathbb{R}$,

$$
|f(\lambda, x, s)-f(\bar{\lambda}, x, s)| \leqslant|\lambda-\bar{\lambda}| K(x) s \quad \forall s \in\left(0, \varepsilon_{0}\right] .
$$

As in $\S 3$, conditions $\left(f_{1}\right),\left(f_{3}^{\prime}\right)$ and $\left(f_{4}\right)$ imply that, if $f_{+}^{\prime}(x, 0) \in L^{r}(\Omega)$ and $\Lambda$ is a bounded set of real numbers, then there exists some positive function $C(x) \in L^{r}(\Omega)$ satisfying

$$
|f(\lambda, x, s)| \leqslant C(x) s \quad \forall s \in\left(0, \varepsilon_{0}\right] \quad \forall \lambda \in \Lambda .
$$

With respect to the matrix $A$, we note that, since its coefficients $a_{i j}(x, s)$ are Carathéodory functions, there exists the limit

$$
\lim _{s \rightarrow 0^{+}} A(x, s)=A(x, 0) \quad \text { a.e. } x \in \Omega .
$$


Observe also that in the case of integrable weight $f_{+}^{\prime}(x, 0)$, the weighted eigenvalue problem

$$
\begin{aligned}
-\operatorname{div}(A(x, 0) \nabla u) & =\lambda f_{+}^{\prime}(x, 0) u & & \text { in } \Omega, \\
u & =0 & & \text { on } \partial \Omega,
\end{aligned}
$$

has a first positive eigenvalue $\lambda_{1}\left(f_{+}^{\prime}(x, 0)\right)$, and it is possible to choose an associated eigenfunction $\varphi>0$ with $\|\varphi\|=1$. We define

$$
\lambda_{0}= \begin{cases}\lambda_{1}\left(f_{+}^{\prime}(x, 0)\right) & \text { if } f_{+}^{\prime}(x, 0) \in L^{r}(\Omega), \\ 0 & \text { otherwise. }\end{cases}
$$

Since we are looking for positive solutions, we can assume, without loss of generality, that

$$
f(\lambda, x, s)=f(\lambda, x, 0)=0 \quad \forall x \in \Omega \quad \forall \lambda \in \mathbb{R} \quad \forall s<0 .
$$

As a consequence, we can apply again the maximum principle to deduce that, for any $\lambda \in \mathbb{R}$, every solution of the equation $u=K(f(\lambda, x, u)), u \in E$, has to be non-negative, and so part (i) of lemma 2.4 remains valid for negative values of $\lambda$.

LEMMA 4.1. Let us suppose that the functions $A(x, s)$ and $f(\lambda, x, s)$ satisfy conditions $\left(A_{1}\right)-\left(A_{3}\right),\left(f_{1}\right),\left(f_{3}^{\prime}\right),\left(f_{4}\right)$ and that $f(\lambda, x, 0)=0$ for a.e. $x \in \Omega$ and for every $\lambda \in \mathbb{R}$. Consider a compact interval $\Lambda \subset\left(-\infty, \lambda_{0}\right)$. Then there exists $\delta>0$ such that

$$
u \neq K(t f(\lambda, x, u))
$$

for every $u \in E$ with $0<\|u\|_{0} \leqslant \delta, \lambda \in \Lambda$ and $t \in[0,1]$.

Proof. We suppose, on the contrary, that there exist sequences $\lambda_{n} \in \Lambda, u_{n} \in E-\{0\}$ and $t_{n} \in[0,1]$ such that $u_{n}=K\left(t_{n} f\left(\lambda_{n}, x, u_{n}\right)\right)$, with $\lambda_{n} \rightarrow \lambda \in \Lambda,\left\|u_{n}\right\|_{0} \rightarrow 0$ and $t_{n} \rightarrow t_{0} \in[0,1]$. Taking $u_{n}$ as a test function and using condition $\left(A_{2}\right)$, we get

$$
\alpha\left\|u_{n}\right\|^{2} \leqslant \int_{\Omega} A\left(x, u_{n}\right) \nabla u_{n} \cdot \nabla u_{n}=t_{n} \int_{\Omega} f\left(\lambda_{n}, x, u_{n}\right) u_{n} .
$$

Observing that, by condition $\left(f_{1}\right), \int_{\Omega} f\left(\lambda_{n}, x, u_{n}\right) u_{n}$ converges to zero, we deduce that $u_{n}$ strongly converges to zero in $H_{0}^{1}(\Omega)$. Taking the normalized sequence $z_{n}:=u_{n}\left\|u_{n}\right\|^{-1}$, we have that $z_{n} \geqslant 0$ and that there exists $z \in H_{0}^{1}(\Omega)$ such that, up to a subsequence, $z_{n}$ weakly converges to $z$ in $H_{0}^{1}(\Omega)$. The normalized sequence $z_{n}$ verifies the following equation:

$$
\int_{\Omega} A\left(x, u_{n}\right) \nabla z_{n} \cdot \nabla v=t_{n} \int_{\Omega} \frac{f\left(\lambda_{n}, x, u_{n}\right)}{\left\|u_{n}\right\|} v \quad \forall v \in H_{0}^{1}(\Omega) .
$$

If $\lambda_{0}=0$, i.e. $f_{+}^{\prime}(x, 0)=+\infty$, then $\Lambda \subset\left(-\infty, \lambda_{0}\right)=(-\infty, 0)$ and, by $\left(f_{4}\right)$, we note that there exists $\varepsilon_{1}>0$ such that $f\left(\lambda_{n}, x, s\right)<0$ a.e. $x \in \Omega$ and for every $0 \leqslant s<\varepsilon_{1}, n \in \mathbb{N}$. Thus, taking $v=z_{n}$ as a test function in (4.4), and using the fact that $\left\|u_{n}\right\|_{0} \leqslant \varepsilon_{1}$ for large $n$, we deduce from $\left(A_{2}\right)$ the following contradiction:

$$
\alpha \leqslant \int_{\Omega} A\left(x, u_{n}\right) \nabla z_{n} \cdot \nabla z_{n}=t_{n} \int_{\Omega} \frac{f\left(\lambda_{n}, x, u_{n}\right)}{\left\|u_{n}\right\|} z_{n} \leqslant 0 .
$$


On the other hand, if $\lambda_{0}>0$, by conditions $\left(A_{1}\right),\left(A_{2}\right)$ and $(4.1)$, following the same arguments as in lemma 3.1, we deduce that $z_{n}$ strongly converges to $z$ in $H_{0}^{1}(\Omega)$. In particular, $z \not \equiv 0$. We conclude this case by observing that $(4.2),\left(f_{3}^{\prime}\right)$, $\left(f_{4}\right)$ and (4.1) allow us to pass to the limit in (4.4), and derive that $z$ verifies

$$
-\operatorname{div}(A(x, 0) \nabla z)=t_{0} \lambda f_{+}^{\prime}(x, 0) z \quad \text { in } \Omega .
$$

Since $z \geqslant 0$ and $z \neq 0$, we get that $z=\phi$ and $t_{0} \lambda=\lambda_{0}$, which is also a contradiction.

REMARK 4.2. As a consequence of lemma 4.1, we deduce that problem $\left(P_{\lambda}\right)$ does not have bifurcation points of positive solutions from zero in $\left(-\infty, \lambda_{0}\right)$. Furthermore, following the same arguments of this lemma, it is also possible to show that there are no other possible bifurcation point from the line of trivial solutions but $\lambda_{0}$. More precisely, we can prove that if $u_{n} \neq 0$ is a solution of problem $\left(P_{\lambda}\right)$ for $\lambda=\lambda_{n}$ with $\lambda_{n} \rightarrow \lambda \neq 0$, and $\left\|u_{n}\right\|_{0} \rightarrow 0$, then $\lambda=\lambda_{0}$. Indeed, as above, the normalized sequence $z_{n}=u_{n} /\left\|u_{n}\right\|$ satisfies (4.4) with $t_{n}=1$ and, without loss of generality, we can assume that $z_{n}$ is weakly convergent to some $z \geqslant 0$ in $H_{0}^{1}(\Omega)$. Now, if $f_{+}^{\prime}(x, 0)$ is integrable, then, as in lemma 4.1 , we deduce that $z \neq 0$ is a solution of the problem

$$
-\operatorname{div}(A(x, 0) \nabla z)=\lambda f_{+}^{\prime}(x, 0) z .
$$

This means that $z=\varphi$ and $\lambda=\lambda_{0}$.

In the case that $f_{+}^{\prime}(x, 0)=+\infty$, consider the eigenvalue problem

$$
-\operatorname{div}\left(A\left(x, u_{n}\right) \nabla v\right)=\mu v, \quad v \in H_{0}^{1}(\Omega),
$$

and let $\phi_{n}>0$ be a normalized eigenfunction associated to the first positive eigenvalue $\mu_{n}$. Recalling that $\mu_{n}$ is variationally characterized as the infimum of

$$
\int_{\Omega} A\left(x, u_{n}\right) \nabla v \cdot \nabla v / \int_{\Omega} v^{2},
$$

for $v \in H_{0}^{1}(\Omega)-\{0\}$, we deduce from $\left(A_{1}\right)$ that, for all $n \in \mathbb{N}, \mu_{n} \leqslant M \equiv \mu \beta$, where $\mu$ denotes the first eigenvalue of the Laplacian operator with zero Dirichlet boundary conditions. Thus, if $\lambda_{n}$ does not converge to $\lambda_{0}=0$, then, passing to a subsequence, we can assume from $\left(f_{4}\right)$ that there exists $n_{0} \in \mathbb{N}$ such that

$$
f\left(\lambda_{n}, x, u_{n}(x)\right)>M u_{n}(x) \quad \text { a.e. } x \in \Omega \quad \forall n \geqslant n_{0} .
$$

Taking $v=\phi_{n}$ as a test function in the equation satisfied by $u_{n}$, we get

$$
\mu_{n} \int_{\Omega} u_{n} \phi_{n}=\int_{\Omega} f\left(\lambda_{n}, x, u_{n}\right) \phi_{n}>M \int_{\Omega} u_{n} \phi_{n} \quad \forall n \geqslant n_{0},
$$

i.e. a contradiction, proving also in this case that $\lambda=\lambda_{0}$.

LEMMA 4.3. Let us assume that $f(\lambda, x, 0)=0$ for a.e. $x \in \Omega$ and for every $\lambda \in \mathbb{R}$, and conditions $\left(A_{1}\right)-\left(A_{3}\right),\left(f_{1}\right),\left(f_{4}\right)$ hold. Consider $\lambda>\lambda_{0}$. Then, for any positive function $\phi$ belonging to $E \cap H_{0}^{1}(\Omega)$, there exists $\delta>0$ such that

$$
u \neq K(f(\lambda, x, u)+\tau \phi),
$$

for every $\tau \geqslant 0$ and for every $u$ in $E$ with $0<\|u\|_{0} \leqslant \delta$. 
Proof. We argue by contradiction and suppose that there exist sequences $\tau_{n} \geqslant 0$, $u_{n} \in E-\{0\}$ with $\left\|u_{n}\right\|_{0} \rightarrow 0$, such that

$$
-\operatorname{div}\left(A\left(x, u_{n}\right) \nabla u_{n}\right)=f\left(\lambda, x, u_{n}\right)+\tau_{n} \phi .
$$

Since $\tau_{n}$ is non-negative, using $u_{n}^{-}$as a test function, we can get, as in the proof of lemma 3.3 , that $u_{n} \geqslant 0$. First, we prove that then $f_{+}^{\prime}(x, 0) \not \equiv+\infty$, i.e. $\lambda_{0}>0$. If not, we follow the same argument of remark 4.2 and we get that (4.5) holds (with $M=\mu \beta \geqslant \mu_{n}$ ). Taking again $v=\phi_{n}$ as a test function in the equation satisfied by $u_{n}$, we get

$\mu_{n} \int_{\Omega} u_{n} \phi_{n}=\int_{\Omega} f\left(\lambda_{n}, x, u_{n}\right) \phi_{n}+\tau_{n} \int_{\Omega} \phi \phi_{n}>M \int_{\Omega} u_{n} \phi_{n}+\tau_{n} \int_{\Omega} \phi \phi_{n} \quad \forall n \geqslant n_{0}$, i.e. a contradiction, proving that $f_{+}^{\prime}(x, 0) \not \equiv+\infty$. Now consider $\phi /\left\|u_{n}\right\|$ as a test function in (4.6) to deduce that the normalized sequence of $u_{n}, z_{n}$ satisfies

$$
\int_{\Omega} A\left(x, u_{n}\right) \nabla z_{n} \cdot \nabla \phi=\int_{\Omega} \frac{f\left(\lambda, x, u_{n}\right)}{\left\|u_{n}\right\|} \phi+\frac{\tau_{n}}{\left\|u_{n}\right\|} \int_{\Omega} \phi^{2} .
$$

In addition, notice that there exists $z$ in $H_{0}^{1}(\Omega)$ such that $z \geqslant 0$ and $z_{n} \rightarrow z$ weakly in $H_{0}^{1}(\Omega)$. Since $(4.1)$ holds, we can use Fatou's lemma to obtain

$$
\begin{aligned}
\int_{\Omega} A(x, 0) \nabla z \cdot \nabla \phi-\limsup _{n \rightarrow \infty} \frac{\tau_{n}}{\left\|u_{n}\right\|} \int_{\Omega} \phi^{2} & \geqslant \liminf _{n \rightarrow \infty} \int_{\Omega} \frac{f\left(\lambda, x, u_{n}\right)}{\left\|u_{n}\right\|} \phi \\
& \geqslant \lambda \int_{\Omega} f_{+}^{\prime}(x, 0) z \phi .
\end{aligned}
$$

Using $\left(A_{1}\right)$ and that $f_{\infty}^{\prime}(x, 0) \in L^{r}(\Omega)$, we derive that

$$
\limsup _{n \rightarrow \infty} \frac{\tau_{n}}{\left\|u_{n}\right\|} \int_{\Omega} \phi^{2} \leqslant \beta \int_{\Omega}|\nabla z \cdot \nabla \phi|-\lambda \int_{\Omega} f_{+}^{\prime}(x, 0) z \phi<+\infty,
$$

and $\tau_{n}\left\|u_{n}\right\|^{-1}$ is bounded. Thus there exists $\tau^{*} \geqslant 0$ such that (up to a subsequence) $\tau_{n}\left\|u_{n}\right\|^{-1} \rightarrow \tau^{*}$. Taking now $\left(z_{n}-z\right) /\left\|u_{n}\right\|$ as a test function in (4.6) and subtracting the integral term $\int_{\Omega} A\left(x, u_{n}\right) \nabla\left(z_{n}-z\right) \cdot \nabla z$, we have, from $\left(A_{2}\right)$,

$\alpha\left\|z_{n}-z\right\|^{2} \leqslant-\int_{\Omega} A\left(x, u_{n}\right) \nabla z \cdot \nabla\left(z_{n}-z\right)+\int_{\Omega} \frac{f\left(\lambda, x, u_{n}\right)}{\left\|u_{n}\right\|}\left(z_{n}-z\right)+\frac{\tau_{n}}{\left\|u_{n}\right\|} \int_{\Omega} \phi\left(z_{n}-z\right)$.

Observing now that, by $\left(A_{1}\right)$ and the Sobolev embedding theorem, we get

$$
\begin{aligned}
\lim _{n \rightarrow \infty} \int_{\Omega} A\left(x, u_{n}\right) \nabla z \cdot \nabla\left(z_{n}-z\right) & =0, \\
\lim _{n \rightarrow \infty} \int_{\Omega} \phi\left(z_{n}-z\right) & =0,
\end{aligned}
$$

and, moreover, by $\left(f_{4}\right)$ and (4.1),

$$
\lim _{n \rightarrow \infty} \int_{\Omega} \frac{f\left(\lambda, x, u_{n}\right)}{\left\|u_{n}\right\|}\left(z_{n}-z\right)=0
$$

we deduce that $z_{n} \rightarrow z$ strongly in $H_{0}^{1}(\Omega)$, so $z \neq 0$. 
Now, divide (4.6) by $\left\|u_{n}\right\|$ and note that conditions $\left(f_{4}\right),(4.1)$ and (4.2) allow us to pass to the limit and obtain that $z$ satisfies the equation

$$
-\operatorname{div}(A(x, 0) \nabla z)=\lambda f_{+}^{\prime}(x, 0) z+\tau^{*} \phi, \quad x \in \Omega .
$$

Let us take $\varphi$ as a test function. Using the fact that $\tau^{*}$ and $\phi$ are positive, we get

$$
\lambda_{0} \int_{\Omega} f_{+}^{\prime}(x, 0) \varphi z=\int_{\Omega} A(x, 0) \nabla z \cdot \nabla \varphi \geqslant \lambda \int_{\Omega} f_{+}^{\prime}(x, 0) \varphi z,
$$

which is a contradiction since $\lambda>\lambda_{0}$.

THEOREM 4.4. Assume that $f(\lambda, x, 0)=0$, for a.e. $x \in \Omega$ and for every $\lambda \in \mathbb{R}$, and that conditions $\left(f_{1}\right),\left(f_{3}^{\prime}\right),\left(f_{4}\right)$ and $\left(A_{1}\right)-\left(A_{3}\right)$ hold. Let $\lambda_{0}$ be given by (4.3). Then $\lambda_{0}$ is a bifurcation point of $\left(P_{\lambda}\right)$ from the trivial solution and it is the only one for positive solutions. Furthermore, there exists an unbounded continuum, i.e. closed and connected subset, $\Sigma_{0}$ in $\Sigma$ meeting $\left(\lambda_{0}, 0\right)$. Furthermore, if $\lambda_{0}>0, r>N$, and (2.2) is satisfied, then the following conclusions hold.

(i) If there exist $\varepsilon_{0}, s_{0}>0, \sigma<0$ and $\underline{C}(x) \in L^{1}(\Omega)$ such that, denoting

$$
\underline{\mu}(x) \equiv \liminf _{(\lambda, s) \rightarrow\left(\lambda_{0}, 0^{+}\right)}\left[f(\lambda, x, s)-\lambda f_{+}^{\prime}(x, 0) s\right] s^{\sigma-1},
$$

for a.e. in $\Omega$, and for all $s \in\left[0, s_{0}\right]$ and $\lambda \in\left(\lambda_{0}-\varepsilon_{0}, \lambda_{0}+\varepsilon_{0}\right)$, we have

$$
\left.\begin{array}{rl}
{[A(x, s)-A(x, 0)]} & \leqslant 0, \\
{\left[f(\lambda, x, s)-\lambda f_{+}^{\prime}(x, 0) s\right] s^{\sigma-1} \geqslant \underline{C}(x),} \\
\int_{\Omega} \underline{\mu}(x) \varphi^{2-\sigma}(x)>0,
\end{array}\right\}
$$

then the bifurcation of positive solutions at $\lambda=\lambda_{0}$ is subcritical.

(ii) If there exist $\varepsilon_{1}, s_{1}>0, \sigma<0$ and $\bar{C}(x) \in L^{1}(\Omega)$ such that, denoting

$$
\bar{\mu}(x) \equiv \limsup _{(\lambda, s) \rightarrow\left(\lambda_{0}, 0^{+}\right)}\left[f(\lambda, x, s)-\lambda f_{+}^{\prime}(x, 0) s\right] s^{\sigma-1},
$$

for a.e. in $\Omega$, and for all $s \in\left[0, s_{1}\right]$ and $\lambda \in\left(\lambda_{0}-\varepsilon_{1}, \lambda_{0}+\varepsilon_{1}\right)$, we have

$$
\begin{aligned}
{[A(x, s)-A(x, 0)] } & \geqslant 0, \\
{\left[f(\lambda, x, s)-\lambda f_{+}^{\prime}(x, 0) s\right] s^{\sigma-1} } & \leqslant \bar{C}(x), \\
\int_{\Omega} \bar{\mu}(x) \varphi^{2-\sigma}(x) & <0,
\end{aligned}
$$

then the bifurcation of positive solutions at $\lambda=\lambda_{0}$ is supercritical.

Proof. First observe that applying lemma 4.1 and following the same argument of theorem 3.4, we get that

$$
\mathrm{i}\left(\Phi_{\lambda}, 0\right)=1 \quad \forall \lambda<\lambda_{0} .
$$

Now let $0<\varepsilon \leqslant \delta$, where $\delta$ is the number given in lemma 4.3. Then, from this lemma and by homotopy invariance,

$$
\operatorname{deg}\left(\Phi_{\lambda}, B_{\varepsilon}, 0\right)=\operatorname{deg}\left(u-K(f(\lambda, x, u)+a \varphi), B_{\varepsilon}, 0\right) \quad \forall a>0 .
$$


Since lemma 4.3 implies also that the problem

$$
u=K(f(\lambda, x, u)+a \varphi), \quad u \in E,
$$

has no solutions in $\bar{B}_{\delta}$, for every $a>0$, we obtain that the degree in the right-hand side is zero, proving that

$$
\mathrm{i}\left(\Phi_{\lambda}, 0\right)=0 \quad \forall \lambda>\lambda_{0}
$$

Then we can follow proposition 4.5 of [1] to conclude the first part of the theorem.

Let us prove assertion (i); the other can be proved similarly. We argue by contradiction and consider a sequence of solutions $\left(\lambda_{n}, u_{n}\right)$ with $\lambda_{n} \geqslant \lambda_{0}, \lambda_{n} \rightarrow \lambda_{0}$, $u_{n} \neq 0$ and $\left\|u_{n}\right\|_{0} \rightarrow 0$. As in theorem 3.4 , by (2.2), we can take as a test function $\varphi^{2}\left(u_{n}\right)^{-1}$ and we get

$$
\begin{aligned}
\int_{\Omega}\left(f\left(\lambda_{n}, x, u_{n}\right)-\lambda_{n} f_{+}^{\prime}(x, 0) u_{n}\right) \frac{\varphi^{2}}{u_{n}} \\
=\int_{\Omega}\left[A\left(x, u_{n}\right)-\frac{\lambda_{n}}{\lambda_{0}} A(x, 0)\right] \nabla \varphi \cdot \nabla \varphi \\
\quad-\int_{\Omega} A\left(x, u_{n}\right)\left(\nabla \varphi-\frac{\varphi}{u_{n}} \nabla u_{n}\right) \cdot\left(\nabla \varphi-\frac{\varphi}{u_{n}} \nabla u_{n}\right) .
\end{aligned}
$$

As before, we denote with $z_{n}$ the normalized sequence of $u_{n}$ and we recall that $z_{n} \rightarrow \varphi$ strongly in $H_{0}^{1}(\Omega)$. Multiplying by $\left\|u_{n}\right\|^{\sigma}$ and applying condition (4.7), we obtain from Fatou's lemma that

$$
0 \geqslant \liminf _{n \rightarrow \infty} \int_{\Omega} \frac{f\left(\lambda_{n}, x, u_{n}\right)-\lambda_{n} f_{+}^{\prime}(x, 0) u_{n}}{u_{n}^{1-\sigma}} z_{n}^{-\sigma} \varphi^{2} \geqslant \int_{\Omega} \underline{\mu}(x) \varphi^{2-\sigma}>0,
$$

which is a contradiction.

REMARK 4.5. The conclusion of theorem 4.4 (ii) is also true in the case $\lambda_{0}=0$, if we impose additional assumptions on $f$. For instance, assuming that, for some $p>1$ and $s_{0}>0$, there exists $K_{1}(x) \in L^{1}(\Omega)$ such that

$$
\left.\begin{array}{ll}
f(0, x, s) \leqslant K_{1}(x) s^{p} & \forall s \in\left[0, s_{0}\right], \\
f(\lambda, x, s) \leqslant f(0, x, s) & \forall \lambda<0, \quad s \in\left[0, s_{0}\right]
\end{array}\right\}
$$

then the bifurcation from $\lambda_{0}=0$ is supercritical. Indeed, we argue by contradiction and assume that there is a sequence of solutions $\left(\lambda_{n}, u_{n}\right)$ with $\lambda_{n} \leqslant 0, \lambda_{n} \rightarrow 0$, $u_{n} \neq 0$ and $\left\|u_{n}\right\|_{0} \rightarrow 0$. Take $u_{n} /\left\|u_{n}\right\|^{2}$ as a test function to get

$$
\int_{\Omega} A\left(x, u_{n}\right) \nabla z_{n} \cdot \nabla z_{n}=\int_{\Omega}\left(f\left(\lambda_{n}, x, u_{n}\right)-f\left(0, x, u_{n}\right)\right) \frac{z_{n}}{\left\|u_{n}\right\|}+\int_{\Omega} f\left(0, x, u_{n}\right) \frac{z_{n}}{\left\|u_{n}\right\|},
$$

where $z_{n}=u_{n} /\left\|u_{n}\right\|$. By conditions $\left(A_{2}\right)$ and (4.8), we obtain from Fatou's lemma that

$$
\alpha \leqslant \limsup _{n \rightarrow \infty} \int_{\left\{u_{n}>0\right\}} \frac{f\left(0, x, u_{n}\right)}{u_{n}} z_{n}^{2} \leqslant 0,
$$


which is a contradiction, proving the assertion. Examples of nonlinearities satisfying the above condition are

$$
\begin{aligned}
& f(\lambda, x, s)=\lambda s^{q}, \quad 0<q \quad(x \in \Omega, s \geqslant 0, \lambda \in \mathbb{R}) \\
& f(\lambda, x, s)=\lambda s^{q}+s^{p}, \quad 0<q \leqslant 1<p \quad(x \in \Omega, s \geqslant 0, \quad \lambda \in \mathbb{R}) .
\end{aligned}
$$

\section{Applications}

In this section we complete the bifurcation results of $\S \S 3$ and 4 by studying the existence or the non-existence of a priori bounds on $\lambda$ or in the norm $\|u\|_{0}$ of solutions $(\lambda, u)$ of $\left(P_{\lambda}\right)$. This will allow us to describe the range of values $\lambda$ for which $\left(P_{\lambda}\right)$ admits at least one solution or at least two solutions. We begin with a result in which both bifurcations, from zero and from infinity, occur.

TheOREM 5.1. Assume that $f(\lambda, x, 0)=0$ for a.e. $x \in \Omega$ and for every $\lambda \in \mathbb{R}$. Suppose also that conditions $\left(A_{1}\right)-\left(A_{4}\right),\left(f_{1}\right)-\left(f_{4}\right)$ and $\left(f_{3}^{\prime}\right)$ hold. Let $\lambda_{\infty}$ and $\lambda_{0}$ be given by (3.2) and (4.3), respectively.

(i) Problem $\left(P_{\lambda}\right)$ has a positive solution for every $\lambda$ between $\lambda_{0}$ and $\lambda_{\infty}$ provided that one of the following conditions is satisfied.

There exists a function $k(x) \in L^{1}(\Omega)$ such that

$f(\lambda, x, s) \geqslant \lambda k(x) s \quad \forall(\lambda, x, s) \in \mathbb{R}^{+} \times \Omega \times \mathbb{R}^{+}, \quad$ with $k^{+} \not \equiv 0$.

There exists $\lambda_{*} \leqslant 0$ such that

$$
f\left(\lambda_{*}, x, s\right) \leqslant 0 \quad \text { a.e. } x \in \Omega \quad \forall s>0,
$$

(ii) If condition (2.2) holds for every $\lambda>0$, and for some $\lambda_{*} \leqslant 0$ and $\bar{s}>0$, we have $\left(f_{6}\right)$ and

$$
f(\lambda, x, \bar{s}) \leqslant 0 \quad \text { a.e. } x \in \Omega \quad \forall \lambda \in\left[\lambda_{*}, \infty\right),
$$

then there exist two solutions for $\lambda>\max \left\{\lambda_{0}, \lambda_{\infty}\right\}$.

REMARK 5.2. In figures 1-3, we give some bifurcation diagrams that may occur in the case (i) for $\lambda_{0}<\lambda_{\infty}$. We note here that the side of the bifurcation is important in order to get the description of the range of values $\lambda$ for which $\left(P_{\lambda}\right)$ has a solution. Figure 1 corresponds to the case in which the hypotheses of theorems 4.4 (ii) and 3.4 (i) are satisfied. Thus the bifurcation is to the right at zero and to the left at infinity and we can only deduce the existence of a solution for $\lambda \in\left(\lambda_{0}, \lambda_{\infty}\right)$. Figure 2 is a bifurcation diagram under assumptions of theorems 4.4 (ii) and 3.4 (ii). In this case, the bifurcation is to the right at zero and at infinity. Hence there exist at least two solutions for $\lambda \in\left(\lambda_{\infty}, \lambda_{\infty}+\varepsilon\right.$ ) (for some $\varepsilon>0$ ) and at least one solution for $\lambda \in\left(\lambda_{0}, \lambda_{\infty}\right)$. On the other hand, if we assume the hypotheses of theorems 4.4 (i) and 3.4 (ii), we obtain the bifurcation diagram given in figure 3 . The bifurcation is to the left at zero and to the right at infinity. In this case, there exist at least two solutions for $\lambda \in\left(\lambda_{0}-\varepsilon_{0}, \lambda_{0}\right) \cup\left(\lambda_{\infty}, \lambda_{\infty}+\varepsilon\right)$ and at least one solution for $\lambda \in\left(\lambda_{0}, \lambda_{\infty}\right)$. Figure 4 is an example of case (ii) of the previous theorem, where the side of the bifurcation is always to the right (for instance, if we assume the hypotheses of theorems 4.4 (ii) and 3.4 (ii)). 


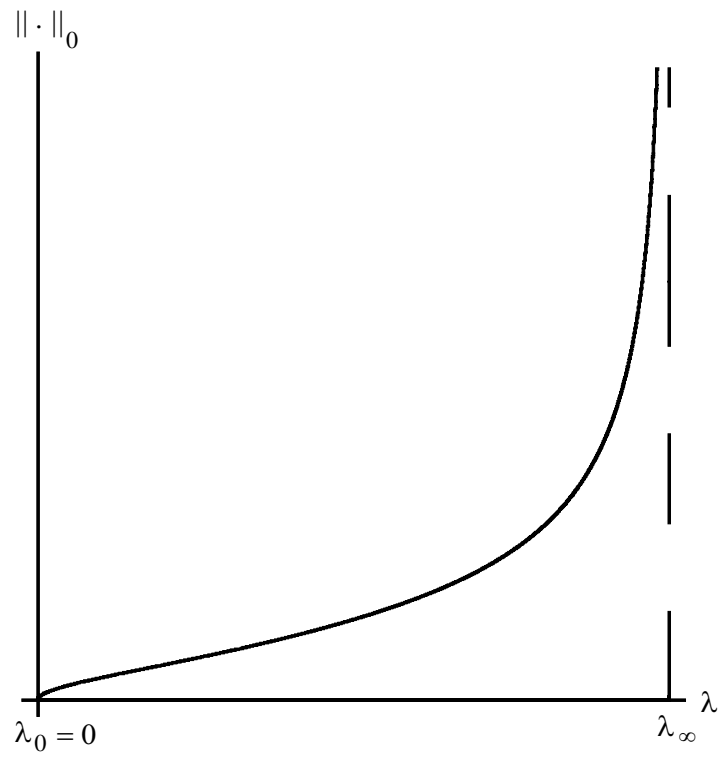

Figure 1.

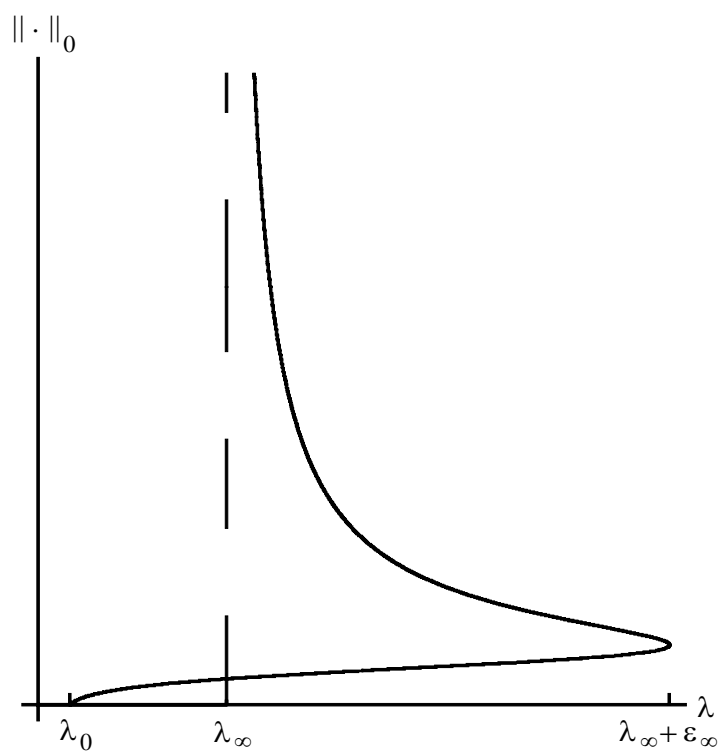

Figure 2.

Proof. Theorems 3.4 and 4.4 prove that there exist two connected sets $\Sigma_{0}$ and $\Sigma_{\infty}$ of positive solutions of $\left(P_{\lambda}\right)$ emanating, respectively, from $\left(\lambda_{0}, 0\right)$ and $\left(\lambda_{\infty}, \infty\right)$, and are the only ones. To prove (i), it suffices to show that there exists either a number $\lambda^{*} \geqslant \max \left\{\lambda_{0}, \lambda_{\infty}\right\}$ such that the equation $\Phi_{\lambda}(u)=0$ has no positive solution with $\lambda=\lambda^{*}$ (this will be true if $\left(f_{5}\right)$ holds), or a number $\lambda_{*} \leqslant \min \left\{\lambda_{0}, \lambda_{\infty}\right\}$ such that $\Phi_{\lambda}(u)=0$ has no positive solution with $\lambda=\lambda_{*}$ (which is true provided $\left(f_{6}\right)$ holds). 


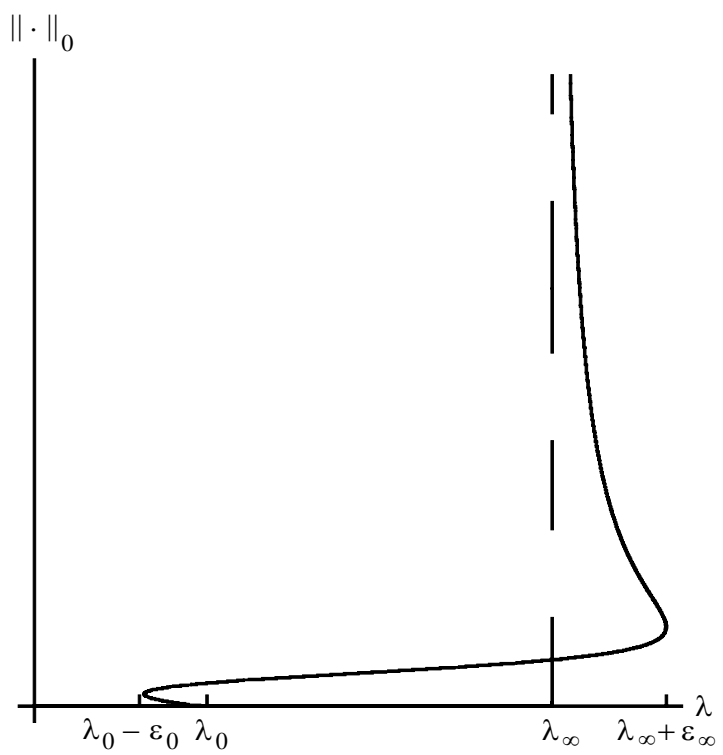

Figure 3.

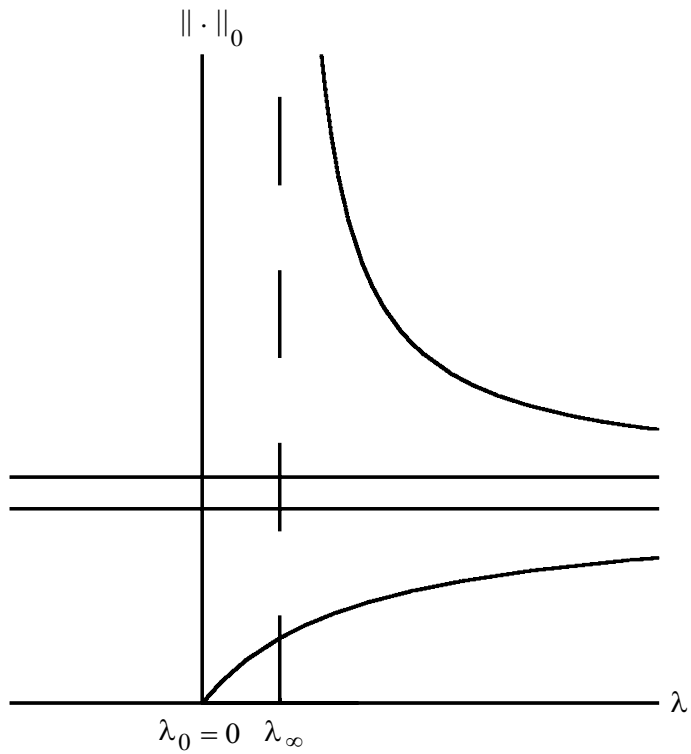

Figure 4 .

Indeed, these facts imply that the projections $\operatorname{Proj} \Sigma_{0}$ and $\operatorname{Proj} \Sigma_{\infty}$ are intervals bounded either from above (by $\lambda^{*}$ ) or from below (by $\lambda_{*}$ ). The global nature of the continua $\Sigma_{0}$ and $\Sigma_{\infty}$ implies that either $\Sigma_{0}=\Sigma_{\infty}$ or, if $\Sigma_{0} \cap \Sigma_{\infty}=\emptyset$, that Proj $\Sigma_{0}$ and Proj $\Sigma_{\infty}$ are unbounded intervals. In either case, the interval of extremes $\lambda_{0}$ and $\lambda_{\infty}$ is contained in Proj $\Sigma_{0} \cup \operatorname{Proj} \Sigma_{\infty}$, proving assertion (i). In order to prove the claim, if $\left(f_{5}\right)$ is satisfied, we take a solution $(\lambda, u)$ of $\Phi_{\lambda}(u)=0$. Since $k^{+} \not \equiv 0$, 
there exists $\psi \in H_{0}^{1}(\Omega),\|\psi\|=1$, such that $\int_{\Omega} k(x) \psi^{2}>0$. By choosing $\varepsilon>0$ and $\psi^{2} /(u+\varepsilon)$ as test functions, we get

$$
\int_{\Omega} A(x, u) \nabla u \cdot\left(2 \frac{\psi}{u+\varepsilon} \nabla \psi-\left(\frac{\psi}{u+\varepsilon}\right)^{2} \nabla(u+\varepsilon)\right)=\int_{\Omega} \frac{f(\lambda, x, u)}{u+\varepsilon} \psi^{2} .
$$

Then, by $\left(A_{1}\right)$,

$$
\begin{aligned}
\beta \geqslant-\int_{\Omega} A(x, u)\left(\nabla \psi-\left(\frac{\psi}{u+\varepsilon}\right) \nabla u\right) \cdot(\nabla \psi & \left.-\left(\frac{\psi}{u+\varepsilon}\right) \nabla u\right) \\
& +\int_{\Omega} A(x, u) \nabla \psi \cdot \nabla \psi \\
= & \int_{\Omega} \frac{f(\lambda, x, u)}{u+\varepsilon} \psi^{2},
\end{aligned}
$$

and since $f(\lambda, x, u) \geqslant \lambda k(x) u$ for $\lambda>0$ and $\int_{\Omega} k(x) \psi^{2}>0$, we deduce that

$$
\beta \geqslant \lambda \int_{\Omega} k(x) \frac{u}{u+\varepsilon} \psi^{2} .
$$

Taking limits as $\varepsilon$ goes to zero yields

$$
\lambda \leqslant \beta / \int_{\Omega} k(x) \psi^{2} .
$$

Therefore, there are no positive solutions of problem $\left(P_{\lambda}\right)$ for $\lambda>\beta / \int_{\Omega} k(x) \psi^{2}$. In particular, since $\lambda_{0}$ and $\lambda_{\infty}$ are bifurcation points of positive solutions, this means that

$$
\beta / \int_{\Omega} k(x) \psi^{2} \geqslant \max \left\{\lambda_{0}, \lambda_{\infty}\right\}
$$

and hence it suffices to choose $\lambda^{*}>\beta / \int_{\Omega} k(x) \psi^{2}$ to obtain the claim in this case.

If $\left(f_{6}\right)$ is satisfied, then there is no positive solution $u$ of $\left(P_{\lambda}\right)$ for $\lambda=\lambda_{*}$. This is easily proved by observing that the unique solution of $\left(P_{\lambda}\right)$ for $\lambda=\lambda_{*}$ is the trivial solution. Indeed, if $u$ is a solution of $\left(P_{\lambda}\right)$ for $\lambda=\lambda_{*}$, then $u \geqslant 0$, and taking $u$ as a test function, we deduce from $\left(A_{2}\right)$ and $\left(f_{6}\right)$ that

$$
\alpha \int_{\Omega}|\nabla u|^{2} \leqslant \int_{\Omega} A(x, u) \nabla u \cdot \nabla u=\int_{\Omega} f\left(\lambda_{*}, x, u\right) u \leqslant 0,
$$

which means that $u \equiv 0$. Finally, as $\lambda_{*} \leqslant 0$, we also have that $\lambda_{*} \leqslant \min \left\{\lambda_{0}, \lambda_{\infty}\right\}$. This concludes the first part of the theorem.

To prove (ii), we recall that $\left(f_{6}\right)$ implies that

$$
\operatorname{Proj} \Sigma_{0}, \operatorname{Proj} \Sigma_{\infty} \subset\left(\lambda_{*},+\infty\right) .
$$

In addition, we claim that for every $\lambda \in \mathbb{R}$ there is no positive solution $u$ of $\left(P_{\lambda}\right)$ with $\|u\|_{0}=\bar{s}$. Indeed, let us argue by contradiction and suppose that there exist $\lambda \in \mathbb{R}$ and a solution $u$ of $\left(P_{\lambda}\right)$ with $\|u\|_{0}=\bar{s}$. Since $f(\lambda, \cdot, \cdot)$ is $C^{1}$ in $\bar{\Omega} \times[0, \infty)$, there exists $m \geqslant 0$ such that $f(\lambda, x, s)+m s$ is increasing in $s \in[0, \bar{s}]$. Then $w=u$ is a solution of the following linear equation:

$$
L(w):=-\operatorname{div}(A(x, u) \nabla w)+m w=f(\lambda, x, u)+m u \quad \text { in } \Omega .
$$


On the other hand, since $f(\lambda, x, \bar{s}) \leqslant 0$, then

$$
L \bar{s} \geqslant f(\lambda, x, \bar{s})+m \bar{s} .
$$

Subtracting the two previous expressions, we get

$$
\begin{aligned}
L(\bar{s}-u) \geqslant 0 & \text { in } \Omega, \\
\bar{s}-u>0 & \text { on } \partial \Omega .
\end{aligned}
$$

Since the coefficients of the matrix $A(x, u)$ are $C^{1}(\bar{\Omega})$-functions, we can deduce from the strong maximum principle that $\bar{s}-u>0$ in $\bar{\Omega}$, which contradicts $\|u\|_{0}=\bar{s}$ and the claim is proved.

In particular, we obtain the inclusions

$$
\Sigma_{0} \subset\left\{(\lambda, u) /\|u\|_{0}<\bar{s}\right\} \quad \text { and } \quad \Sigma_{\infty} \subset\left\{(\lambda, u) /\|u\|_{0}>\bar{s}\right\},
$$

and, moreover, $\Sigma_{0} \cap \Sigma_{\infty}=\emptyset$. Then we conclude the proof by observing that, by the global nature of the bifurcations, we get that $\left[\lambda_{0},+\infty\right) \subset \operatorname{Proj} \Sigma_{0}$ and $\left(\lambda_{\infty},+\infty\right) \subset \operatorname{Proj} \Sigma_{\infty}$, which implies that, for every $\lambda>\max \left\{\lambda_{0}, \lambda_{\infty}\right\}$, we have two positive solutions of $\left(P_{\lambda}\right)$, one in $\Sigma_{0}$ and the other in $\Sigma_{\infty}$.

REMARK 5.3. Theorem 5.1 extends to the case of quasilinear operators in $[1$, theorem A]. Moreover, applying theorem 5.1, it is also possible to handle nonlinearities that have different behaviour near the origin and near infinity.

REMARK 5.4. We note also that if both conditions $\left(f_{6}\right)$ and $\left(f_{7}\right)$ are satisfied, then the continua $\Sigma_{0}, \Sigma_{\infty}$ emanating, respectively, from zero and from infinity are the same set, i.e. $\Sigma_{0}=\Sigma_{\infty}$.

TheOREm 5.5. Let $\left(A_{1}\right)-\left(A_{4}\right),\left(f_{1}\right),\left(f_{3}\right),\left(f_{4}\right),\left(f_{3}^{\prime}\right)$ and $\left(f_{6}\right)$ be satisfied. Assume that $f(\lambda, x, 0)=0$ a.e. $x \in \Omega$ and for every $\lambda \in \mathbb{R}$, and suppose that

$$
\lim _{s \rightarrow+\infty} \frac{f(\lambda, x, s)}{s}=0, \text { uniformly for } x \in \Omega \quad \forall \lambda \geqslant \lambda_{*},
$$

where $\lambda_{*}$ is given in $\left(f_{6}\right)$. Then there exists at least a positive solution of $\left(P_{\lambda}\right)$ for every $\lambda \geqslant \lambda_{0}$.

REMARK 5.6. Under the hypotheses of the above theorem, if $\lambda_{0}=0$, then the bifurcation diagram will be as in figure 5 .

Proof. Thanks to theorem 4.4, there exists a bifurcation from zero at $\lambda_{0}$. In the proof of theorem 5.1, we have seen that condition $\left(f_{6}\right)$ implies the non-existence of positive solutions of $\left(P_{\lambda}\right)$ for $\lambda=\lambda_{*}$. To conclude, it suffices to prove that there is no bifurcation point from infinity. In other words, we only have to prove the a priori bound for solutions of $\left(P_{\lambda}\right)$ in bounded sets of $\lambda$. Let us argue by contradiction, so let $\lambda_{n} \rightarrow \lambda<+\infty$ and let $u_{n}$ be a non-trivial solution of $\left(P_{\lambda}\right)$ for $\lambda=\lambda_{*}$ with $\left\|u_{n}\right\|_{0} \rightarrow+\infty$. Without loss of generality, we can assume that $z_{n} \equiv u_{n} /\left\|u_{n}\right\| \rightarrow z$ strongly in $L^{2}(\Omega)$. Then, taking $u_{n} /\left\|u_{n}\right\|^{2}$ as a test function, we obtain that $z_{n}$ satisfies

$$
\int_{\Omega} A\left(x, u_{n}\right) \nabla z_{n} \cdot \nabla z_{n}=\int_{\Omega} f\left(\lambda_{n}, x, u_{n}\right) \frac{z_{n}}{\left\|u_{n}\right\|} .
$$




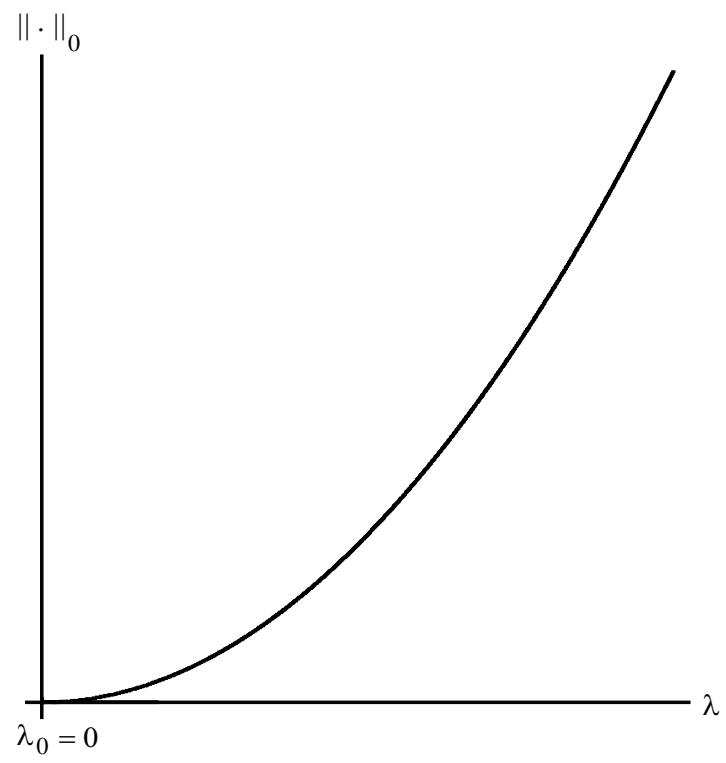

Figure 5.

Now, we get from conditions $\left(A_{2}\right)$ and $\left(f_{3}\right)$ that

$$
\begin{aligned}
& \alpha \leqslant \int_{\Omega} \frac{f\left(\lambda_{n}, x, u_{n}\right)}{\left\|u_{n}\right\|} z_{n} \\
& \leqslant\left|\lambda_{n}-\lambda\right| \int_{\Omega}\left(K_{1}(x) z_{n}+\frac{K_{2}(x)}{\left\|u_{n}\right\|}\right) z_{n} \\
& \quad+\int_{\Omega} \frac{f\left(\lambda, x, u_{n}\right)}{\left\|u_{n}\right\|} z+\int_{\Omega} \frac{f\left(\lambda, x, u_{n}\right)}{\left\|u_{n}\right\|}\left(z_{n}-z\right) .
\end{aligned}
$$

Condition (5.1) implies that

$$
\frac{f\left(\lambda, x, u_{n}\right)}{u_{n}} \rightarrow 0
$$

almost everywhere in $\Omega^{+}:=\{x \in \Omega / z(x)>0\}$. Thus, by condition (3.1) (which derives from $\left(f_{1}\right),\left(f_{3}\right)$ and $\left.(5.1)\right)$, and the Lebesgue dominated convergence theorem, we obtain that

$$
\lim _{n \rightarrow \infty} \int_{\Omega} f\left(\lambda, x, u_{n}\right) \frac{z}{\left\|u_{n}\right\|}=\lim _{n \rightarrow \infty} \int_{\Omega^{+}} \frac{f\left(\lambda, x, u_{n}\right)}{u_{n}+1}\left(z z_{n}+\frac{z}{\left\|u_{n}\right\|}\right)=0 .
$$

Since $z_{n}$ converges to $z$, and by (3.1), we have

$$
\lim _{n \rightarrow+\infty} \int_{\Omega} \frac{f\left(\lambda, x, u_{n}\right)}{\left\|u_{n}\right\|}\left(z_{n}-z\right)=0 .
$$

Then, as $\lambda_{n} \rightarrow \lambda$, we get a contradiction with the fact that $\alpha>0$.

REMARK 5.7. In [7], the method of sub and supersolutions is used to prove the existence of at least one positive solution of $\left(P_{\lambda}\right)$ for every $\lambda>0$ for the nonlinearity 
$f(\lambda, x, s)=\lambda s^{q}, 0<q<1$. Note that this result is a particular case of our theorem with $f_{+}^{\prime}(x, 0)=+\infty\left(\lambda_{0}=0\right)$, with the additional information that there is a solution lying in the continuum emanating from zero at $\lambda_{0}=0$. In addition, our theorem also covers cases in which $f_{+}^{\prime}(x, 0)<+\infty$.

In the semilinear case, i.e. $A(x, s)=A(x)$, the uniqueness of positive solutions of $\left(P_{\lambda}\right)$ for $\lambda>0$ and concave nonlinearities $f(\lambda, x, s)$ in the variable $s$ is well known [10] (see also [9] for interesting different proofs of the uniqueness). With respect to the extension of this uniqueness result to general coefficients $A(x, s)$, the following result holds.

THEOREM 5.8. Let $\left(A_{1}\right)-\left(A_{3}\right)$ and (2.2) be satisfied and assume that there exists $\varepsilon_{0} \in(0,+\infty]$ such that

$$
A(x, s) \text { is non-decreasing for with respect to } s, \quad 0 \leqslant s<\varepsilon_{0} \text {. }
$$

Also let $\lambda>0$ be fixed. Assume $\left(f_{1}\right)$ and

$$
\begin{gathered}
\frac{f(\lambda, x, s)}{s} \text { is decreasing for } 0 \leqslant s<\varepsilon_{0} \quad \text { a.e. } x \in \Omega, \\
f(\lambda, x, s) \geqslant \beta \mu_{1} s \quad \text { a.e. } x \in \Omega, \quad s \in\left[0, \varepsilon_{0}\right]
\end{gathered}
$$

where $\mu_{1}$ denotes the first eigenvalue of the Laplacian operator. Then problem $\left(P_{\lambda}\right)$ has at most one positive solution $u$ with $\|u\|_{0}<\varepsilon_{0}$.

REMARK 5.9. If we assume the following stronger hypotheses,

$$
\begin{aligned}
& A(x, s) \text { is non-decreasing with respect to } s \text { for } 0 \leqslant s<+\infty \text { a.e. } x \in \Omega, \\
& \qquad \frac{f(\lambda, x, s)}{s} \text { is decreasing for } 0 \leqslant s<+\infty \text { a.e. } x \in \Omega, \\
& \qquad \lim _{s \rightarrow 0^{+}} \frac{f(\lambda, x, s)}{s}=+\infty \text {, uniformly in } \Omega,
\end{aligned}
$$

then theorem 5.8 implies uniqueness of positive solutions of problem $\left(P_{\lambda}\right)$.

REMARK 5.10. Some words are in order with respect to condition $\left(A_{5}\right)$. In the case that $A(x, s)=a(s) I$ and $f(\lambda, x, s)=\lambda f(s)$, performing the change of variable $v=\hat{a}(u)$, where

$$
\hat{a}(s)=\int_{0}^{s} a(t) \mathrm{d} t,
$$

we get that $\left(P_{\lambda}\right)$ is equivalent to the following semilinear boundary-value problem:

$$
\begin{aligned}
-\Delta v(x) & =\lambda\left(f \circ \hat{a}^{-1}\right)(v(x)), & & x \in \Omega, \\
v(x) & =0, & & x \in \partial \Omega .
\end{aligned}
$$

Uniqueness theorems are known (see [10]) if the composed function $f \circ \hat{a}^{-1}$ is concave. This is so when hypotheses $\left(A_{5}\right)$ and (5.2) are satisfied in this case.

Proof. We argue by contradiction and suppose that $u_{1}$ and $u_{2}$ are distinct solutions of $\left(P_{\lambda}\right)$ with $\left\|u_{i}\right\|_{0}<\varepsilon_{0}, i=1,2$. We claim that it is then possible to choose $u_{1}$ 
and $u_{2}$ such that $u_{1} \leqslant u_{2}$. Indeed, let us recall that in [8] it is proved that, for each $r>0$, there exist $u_{r} \in \dot{P} \subset E$ and $\lambda_{r} \in \mathbb{R}^{+}$such that $\left\|u_{r}\right\|_{r}=r$ and

$$
\begin{aligned}
-\operatorname{div}\left(A\left(x, u_{r}\right) \nabla u_{r}\right) & =\lambda_{r} u_{r}, & & x \in \Omega, \\
u_{r} & =0, & & x \in \partial \Omega .
\end{aligned}
$$

Moreover, we have $\left\|u_{r}\right\|_{0} \leqslant c_{0} \lambda_{r} r$. Following the same arguments of [7], by (5.3), for sufficiently small $r>0, u_{r}$ is a subsolution of $\left(P_{\lambda}\right)$. Since, by lemma 2.4 (ii), $u_{1}$ and $u_{2}$ are in $\dot{P}$, we have, for $r$ sufficiently small, that $u_{r} \leqslant \min \left\{u_{1}, u_{2}\right\}$. Thus, by the sub-supersolutions method (see [7]), there exists a solution $w_{1}$ of $\left(P_{\lambda}\right)$ such that

$$
u_{r} \leqslant w_{1} \leqslant u_{1} \quad \text { and } \quad u_{r} \leqslant w_{1} \leqslant u_{2} .
$$

Hence we have obtained two ordered distinct solutions: either $w_{1}$ and $u_{1}$, or $w_{1}$ and $u_{2}$. Therefore, we can always assume $u_{1} \leqslant u_{2}<\varepsilon_{0}$, and $u_{1} \not \equiv u_{2}$. We take $u_{2}^{2} / u_{1}$ as a test function in the equation satisfied by $u_{1}$ and we get

$$
\int_{\Omega} A\left(x, u_{1}\right) \nabla u_{1} \cdot\left(2 \frac{u_{2}}{u_{1}} \nabla u_{2}-\left(\frac{u_{2}}{u_{1}}\right)^{2} \nabla u_{1}\right)=\int_{\Omega} \frac{f\left(\lambda, x, u_{1}\right)}{u_{1}} u_{2}^{2} .
$$

Now taking $u_{2}$ as a test function in the equation satisfied by $u_{2}$ yields

$$
\int_{\Omega} A\left(x, u_{2}\right) \nabla u_{2} \cdot \nabla u_{2}=\int_{\Omega} \frac{f\left(\lambda, x, u_{2}\right)}{u_{2}} u_{2}^{2} .
$$

Observing that $u_{1} \leqslant u_{2}<\varepsilon_{0}$, we deduce from $\left(A_{5}\right)$ that $A\left(x, u_{2}\right)-A\left(x, u_{1}\right)$ is non-negative definite and, subtracting both equalities, we obtain from (5.2) that

$$
\begin{aligned}
0 \geqslant-\int_{\Omega} A\left(x, u_{1}\right)\left(\nabla u_{2}-\frac{u_{2}}{u_{1}} \nabla u_{1}\right) \cdot\left(\nabla u_{2}-\frac{u_{2}}{u_{1}} \nabla u_{1}\right) \\
\quad-\int_{\Omega}\left[A\left(x, u_{2}\right)-A\left(x, u_{1}\right)\right] \nabla u_{2} \cdot \nabla u_{2} \\
=\int_{\Omega}\left(\frac{f\left(\lambda, x, u_{1}\right)}{u_{1}}-\frac{f\left(\lambda, x, u_{2}\right)}{u_{2}}\right) u_{2}^{2}>0,
\end{aligned}
$$

which is a contradiction.

Now let us deal with nonlinearities $f$ that are superlinear at infinity. Specifically, we study the case in which there exists $p \in\left(1,2^{*}-1\right)$ and a bounded function $h$ such that, for every real compact interval $\Lambda$,

$$
\lim _{s \rightarrow+\infty} \frac{f(\lambda, x, s)}{s^{p}}=h(x) \geqslant c>0, \text { uniformly, } \quad x \in \Omega, \quad \lambda \in \Lambda .
$$

The following extension of the a priori estimate of Gidas and Spruck [16] to quasilinear operators will be useful.

THEOREM 5.11. Let us assume that $a_{i j}(x, s) \in C^{1}(\bar{\Omega} \times \mathbb{R})$ and conditions $\left(A_{1}\right)$, $\left(A_{2}\right),\left(A_{4}\right),\left(f_{1}\right)$ and (5.7) hold. Then, for every compact interval $\Lambda \subset \mathbb{R}$, there exists $C>0$ such that every positive solution $u \in C^{1}(\Omega) \cap C^{0}(\bar{\Omega})$ of $\left(P_{\lambda}\right)$ with $\lambda \in \Lambda$ satisfies

$$
u(x) \leqslant C \quad \forall x \in \Omega .
$$


Proof. The proof follows the outline of [16], with a few elementary changes. However, in order to make the paper as self-contained as possible, we include the details here. Let us denote by $\mathcal{B}_{R}(x)$ a ball in $\mathbb{R}^{N}$ centred at $x \in \mathbb{R}^{N}$ of radius $R>0$. We argue by contradiction and suppose that $u_{n} \in C^{1}(\Omega) \cap C^{0}(\bar{\Omega})$ are positive solutions of $\left(P_{\lambda}\right)$ for $\lambda=\lambda_{*}$, with $\lambda_{n} \in \Lambda$ and $P_{n} \in \Omega$, such that

$$
M_{n}=\sup _{\Omega} u_{n}(x)=u_{n}\left(P_{n}\right) \rightarrow+\infty .
$$

Then (up to a subsequence) we may assume

$$
\lambda_{n} \rightarrow \lambda, \quad P_{n} \rightarrow P \in \bar{\Omega} .
$$

We divide the proof into two steps, in the first one we suppose that $P \in \Omega$, while in the second one we deal with the case in which $P \in \partial \Omega$. In both cases, we will obtain a contradiction.

SteP 1. Suppose that $P \in \Omega$. Let $2 d=\operatorname{dist}(P, \partial \Omega)>0, \mu_{n}=M_{n}^{(1-p) / 2}$ and

$$
v_{n}(y)=\mu_{n}^{2 /(p-1)} u_{n}\left(P_{n}+\mu_{n} y\right) \quad \text { for every } y \in \mathcal{B}_{d / \mu_{n}}(0) .
$$

Observe that $\mu_{n} \rightarrow 0$,

$$
\sup _{y \in \mathcal{B}_{d / \mu_{n}}(0)} v_{n}(y)=v_{n}(0)=1,
$$

and $v_{n}$ satisfies the following equation in $\mathcal{B}_{d / \mu_{n}}(0)$ :

$$
\begin{aligned}
& -\operatorname{div}\left(A\left(\mu_{n} y+P_{n}, \mu_{n}^{-2 /(p-1)} v_{n}(y)\right) \nabla v_{n}\right) \\
& \quad=\mu_{n}^{2 p /(p-1)} f\left(\lambda_{n}, \mu_{n} y+P_{n}, \mu_{n}^{-2 /(p-1)} v_{n}(y)\right) .
\end{aligned}
$$

By (5.7), the right-hand side of this equation satisfies

$$
\lim _{n \rightarrow \infty}\left|\mu_{n}^{2 p /(p-1)} f\left(\lambda_{n}, \mu_{n} y+P_{n}, \mu_{n}^{-2 /(p-1)} v_{n}(y)\right)-h\left(\mu_{n} y+P_{n}\right) v_{n}(y)^{p}\right|=0 .
$$

Since conditions $\left(A_{1}\right),\left(A_{2}\right)$ hold, by applying theorem 9.15 in [17], we get that $v_{n} \in W^{2, s}\left(\mathcal{B}_{d / \mu_{n}}(0)\right), s>1$. Now fix $R>0$ and let $n_{0}$ be a positive integer such that $R<d / \mu_{n}$ for every $n \geqslant n_{0}$. For every $R^{\prime} \in\left(R, d / \mu_{n}\right)$, we obtain from theorem 9.11 in [17] that

$$
\left\|v_{n}\right\|_{W^{2, s}\left(\mathcal{B}_{R}(0)\right)} \leqslant C\left(\left\|v_{n}\right\|_{L^{s}\left(\mathcal{B}_{R^{\prime}}(0)\right)}+\left\|g_{n}\right\|_{L^{s}\left(\mathcal{B}_{R^{\prime}}(0)\right)}\right),
$$

where $C$ is a positive constant depending only on $N, p, \alpha, \beta$ and $R^{\prime}$, and

$$
g_{n}(y)=\mu_{n}^{2 p /(p-1)} f\left(\lambda_{n}, \mu_{n} y+P_{n}, \mu_{n}^{-2 /(p-1)} v_{n}(y)\right) .
$$

Taking into account that

$$
\left\|v_{n}\right\|_{L^{s}\left(\mathcal{B}_{R^{\prime}}(0)\right)}+\left\|g_{n}\right\|_{L^{s}\left(\mathcal{B}_{R^{\prime}}(0)\right)} \leqslant C_{1}=C_{1}\left(R^{\prime}, \lambda_{n}\right) \quad \forall n \geqslant n_{0},
$$

we deduce a uniform bound for $\left\|v_{n}\right\|_{W^{2, s}\left(\mathcal{B}_{R}(0)\right)}$ for every $n \geqslant n_{0}$. Choosing $s$ large enough, we obtain from Morrey's theorem that $\left\|v_{n}\right\|_{C^{1, \beta}\left(\overline{\mathcal{B}_{R}(0)}\right)}$ is uniformly bounded. Therefore, we can apply the Ascoli-Arzelá theorem and deduce the existence of a function $v \in C^{0}\left(\overline{\mathcal{B}_{R}(0)}\right)$ such that (up to a subsequence) $v_{n} \rightarrow v$ in 
$C^{0}\left(\overline{\mathcal{B}_{R}(0)}\right)$ and $h\left(\mu_{n} y+P_{n}\right) \rightarrow \nu$, for some $\nu>0$. Necessarily, we have that $v(0)=1$ and, from $\left(A_{4}\right)$,

$$
-\operatorname{div}(A(P,+\infty) \nabla v)=\nu v^{p}
$$

Then, by regularity, for $\tau \in(0,1), v \in C^{1, \tau}\left(\overline{\mathcal{B}_{R}(0)}\right)$. From the arbitrariness of $R>0$, we deduce that $v$ is defined in $\mathbb{R}^{N}$ and it is a solution, after rotating and stretching coordinates, of

$$
\begin{aligned}
-\Delta v(x) & =v^{p}(x), \quad x \in \mathbb{R}^{N}, \\
v(0) & =1 .
\end{aligned}
$$

This contradicts theorem 1.2 in [16].

Step 2. Suppose now that $P \in \partial \Omega$. In this case, since $\partial \Omega$ is smooth, we can suppose that a neighbourhood of $P$ in $\Omega$ is contained in the set $\left\{x \in \mathbb{R}^{N}: x_{N}>0\right\}$ and that near $P$ the boundary of $\Omega$ is contained in the hyperplane $x_{N}=0$. We set $d_{n}=\operatorname{dist}\left(P_{n}, \partial \Omega\right)=P_{n} \cdot e_{n}\left(e_{n}=(0, \ldots, 0,1)\right)$, and we observe that the function $v_{n}$ given by (5.8) is well defined in $\Omega_{n} \equiv \mathcal{B}_{\delta / \mu_{n}}(0) \cap\left\{y_{N}>-d_{n} / \mu_{n}\right\}$, for some $\delta>0$. Moreover, it satisfies (5.9) in $\Omega_{n}$. By elliptic regularity up to the boundary (see [17, theorems 9.13 and 9.15]) and Morrey's theorem, we deduce, again from $\left(A_{1}\right),\left(A_{2}\right)$, that $\left|\nabla v_{n}\right|$ is uniformly bounded in $\Omega_{n}$. Consequently,

$$
1=\left|v_{n}(0)-v_{n}\left(-\frac{d_{n}}{\mu_{n}} e_{n}\right)\right| \leqslant C \frac{d_{n}}{\mu_{n}},
$$

i.e. $d_{n} / \mu_{n}$ is away from zero. If, for a subsequence, $d_{n} / \mu_{n} \rightarrow \infty$, we can apply similar arguments to those of step 1 to reach a contradiction with theorem 1.2 in [16]. On the other hand, if $d_{n} / \mu_{n}$ is bounded from above, we assume, passing to a subsequence if necessary, that $d_{n} / \mu_{n} \rightarrow s>0$. Since $v_{n}$ satisfies (5.9) in $\Omega_{n}$, again by [17, theorem 9.15], for every $R, \varepsilon>0$, we get a uniform bound of $v_{n}$ in $C^{1, \tau}\left(\mathcal{B}_{R}(0) \cap\left\{y_{N}>-s+\varepsilon\right\}\right)$ for $n$ large enough. Therefore (up to a subsequence), we obtain that $v_{n} \rightarrow v$ in $C^{1}\left(\mathcal{B}_{R}(0) \cap\left\{y_{N}>-s+\varepsilon\right\}\right), h\left(\mu_{n} y+P_{n}\right) \rightarrow \nu$, for some $\nu>0$, and, using $\left(A_{4}\right)$ and that $R$ and $\varepsilon$ are arbitrary, $v$ is a solution of

$$
\begin{aligned}
-\operatorname{div}(A(P,+\infty) \nabla v) & =\nu v^{p}, & & \left\{y_{N}>-s\right\}, \\
v(y) & =0, & & \left\{y_{N}=-s\right\}, \\
v(0) & =1 . & &
\end{aligned}
$$

Now the contradiction follows from theorem 1.3 in [16] after a linear transformation and a stretching of coordinates.

We are now ready to state our existence result.

TheOREM 5.12. Assume (2.2) and that $f(\lambda, x, 0)=0$, for a.e. $x \in \Omega$ and for every $\lambda \in \mathbb{R},\left(f_{1}\right),\left(f_{3}^{\prime}\right),\left(f_{4}\right),\left(f_{5}\right),(5.7)$ and $\left(A_{1}\right)-\left(A_{4}\right)$. Let $\lambda_{0}$ be given by (4.3). Then there exist $\Lambda^{*} \geqslant \bar{\lambda} \geqslant \lambda_{0}$ such that $\left(P_{\lambda}\right)$ has at least one positive solution for every $\lambda \leqslant \bar{\lambda}$ and no positive solution if $\lambda>\Lambda^{*}$. In addition, if either $\lambda_{0}>0$ and the hypotheses of theorem 4.4 (ii) hold, or $\lambda_{0}=0$ (i.e. $f_{+}^{\prime}(x, 0)=+\infty$, for every $x \in \Omega$ ) and (4.8) is satisfied, then $\bar{\lambda}>\lambda_{0}$ and, for every $\lambda \in\left(\lambda_{0}, \bar{\lambda}\right)$, there exist at least two positive solutions. 


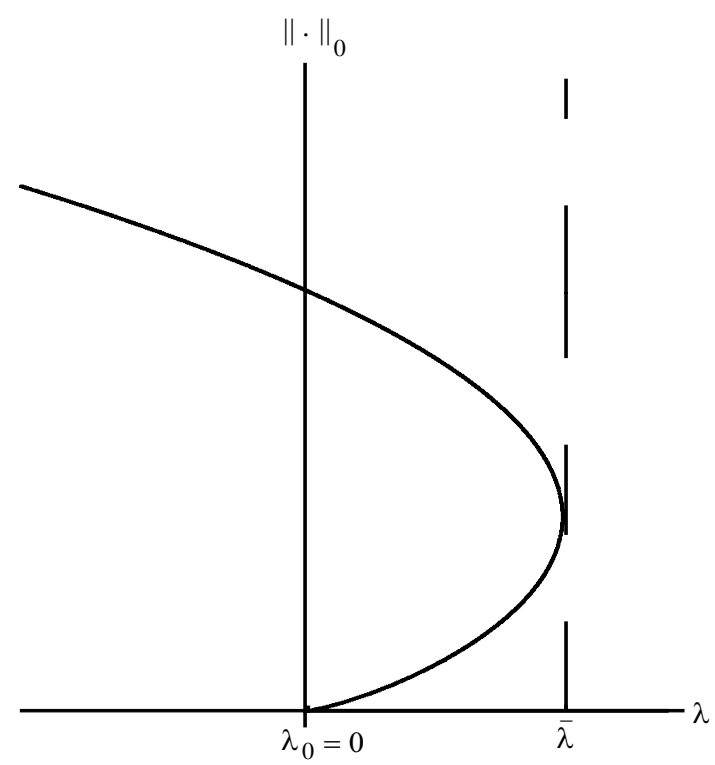

Figure 6 .

REMARK 5.13. Under the hypotheses of theorem 5.12, once again, we see that the study of the side of the bifurcation from $\left(\lambda_{0}, 0\right)$ is essential. If it occurs to the left (for instance, if $\lambda_{0}>0$ and the hypotheses of theorem 4.4 (i) hold), only existence of at least one positive solution for $\lambda<\lambda_{0}$ is deduced. On the other hand, if it is to the right (e.g. if either $\lambda_{0}>0$ and the hypotheses of theorem 4.4 (ii) are satisfied or $\lambda_{0}=0$ and (4.8) holds), then $\bar{\lambda}>\lambda_{0}$ and we obtain the existence of at least two positive solutions for $\lambda$ in a right-hand side interval of $\lambda_{0}$ (see figure 6).

Proof. Since the hypotheses of theorem 4.4 are satisfied, from $\lambda_{0}$ emanates an unbounded continuum $\Sigma_{0} \subset \Sigma$. By $\left(f_{5}\right)$, as we have seen in the proof of theorem 5.1 (i), there is an a priori upper bound for the set of values $\lambda$ for which there exists a solution of $\left(P_{\lambda}\right)$. Thus

$$
\Lambda^{*} \equiv \sup \left\{\lambda \in \mathbb{R} ; \exists u \in E-\{0\} \text { solution of }\left(P_{\lambda}\right)\right\}<+\infty,
$$

and $\operatorname{Proj} \Sigma_{0}$ is a real interval bounded from above by $\Lambda^{*}$. Hence, if $\bar{\lambda}$ is the supremum of Proj $\Sigma_{0}$, then $\lambda_{0} \in \operatorname{Proj} \Sigma_{0} \subset(-\infty, \bar{\lambda}]$. Using theorem 5.11 and the fact that $\Sigma_{0}$ is unbounded, we also deduce that $(-\infty, \bar{\lambda}] \subset \operatorname{Proj} \Sigma_{0}$. This proves the first part of the theorem. If either $\lambda_{0} \neq 0$ and the hypotheses of theorem 4.4 (ii) hold, or $\lambda_{0}=0$ and (4.8) is satisfied, then the bifurcation is supercritical and so $\lambda_{0}<\bar{\lambda}$. Therefore, we get the existence of at least two positive solutions for every $\lambda \in\left(\lambda_{0}, \bar{\lambda}\right)$.

A question remains open in theorem 5.12. Indeed, it is natural to wonder whether the continuum $\Sigma_{0}$ gives the maximal interval of $\lambda$ for which there exists a positive solution of $\left(P_{\lambda}\right)$, i.e. is it $\bar{\lambda}=\Lambda^{*}$ ? We can prove that the answer is positive for a particular class of matrices $A(x, s)$. Specifically, we assume that

$$
A(x, s):=a(s) A(x) \quad(i, j=1, \ldots N),
$$


where $A(x):=\left(a_{i j}(x)\right)$ is a positive definite matrix with $C^{1}$-coefficients $a_{i j}(x)$ in $\bar{\Omega}$ and $a:[0,+\infty) \rightarrow \mathbb{R}$ a non-increasing $C^{1}$-function with $\lim _{s \rightarrow+\infty} a(s)>0$. Note, that in this case, conditions $\left(A_{1}\right)-\left(A_{3}\right)$ hold. With respect to $f(\lambda, x, s)$, we assume that $f(\lambda, x, 0)=0$ a.e. $x \in \Omega$, for every $\lambda \in \mathbb{R}$ and $f$ is non-decreasing in $s$. We need to prove the following theorem.

TheOREM 5.14. Assume that $\left(A_{6}\right)$ holds, that $f(\lambda, x, 0)=0$ a.e. $x \in \Omega$, for every $\lambda \in \mathbb{R}$ and that $f$ is non-decreasing in $s$. Let $I \subset \mathbb{R}$ be an interval, and $\Sigma_{0} \subset$ $I \times C_{0}^{1}(\bar{\Omega})$ be a connected set of solutions of $\left(P_{\lambda}\right)$. Consider a continuous map $T: I \rightarrow C_{0}^{1}(\bar{\Omega})$ such that $T(\lambda)$ is a supersolution of $\left(P_{\lambda}\right)$, which is not a solution. If there exists $\left(\lambda_{0}, u_{0}\right) \in \Sigma_{0}$ with $u_{0} \leqslant T\left(\lambda_{0}\right)$ in $\Omega$, then $u \leqslant T(\lambda)$ in $\Omega \forall(\lambda, u) \in \Sigma_{0}$.

Proof. This theorem is an extension to quasilinear operators of the result in [15, theorem 2.2]. Arguing in the same way as [15], we note that it is sufficient to show the following extension of lemma 2.1 of [15].

LEMmA 5.15. Let $\bar{u} \in C_{0}^{1}(\bar{\Omega})$ be a supersolution of $\left(P_{\lambda}\right)$ and $u \in C_{0}^{1}(\bar{\Omega})$ be a solution of $\left(P_{\lambda}\right)$ such that $u \neq \bar{u}$. Then $\bar{u}-u \notin \partial P$.

Proof. Let $\hat{a}:[0,+\infty) \rightarrow \mathbb{R}$ be defined by

$$
\hat{a}(s)=\int_{0}^{s} a(t) \mathrm{d} t .
$$

The function $\hat{a}$ is increasing and $\hat{a}(0)=0$. In addition, by the chain rule, $u$ is a positive solution of $\left(P_{\lambda}\right)$, i.e.

$$
\int_{\Omega} a(u) A(x) \nabla u \cdot \nabla v=\int_{\Omega} f(\lambda, x, u) v \quad \forall v \in H_{0}^{1}(\Omega)
$$

if and only if $w=\hat{a}(u)$ satisfies

$$
\int_{\Omega} A(x) \nabla \hat{a}(u) \cdot \nabla v=\int_{\Omega} f(\lambda, x, u) v \quad \forall v \in H_{0}^{1}(\Omega),
$$

i.e. $w=\hat{a}(u)$ verifies

$$
\begin{aligned}
-\operatorname{div}(A(x) \nabla w) & =f(\lambda, x, u) \geqslant 0, & & x \in \Omega, \\
w & \geqslant 0, & & x \in \Omega, \\
w & =0, & & x \in \partial \Omega .
\end{aligned}
$$

Notice that, in this case, by the maximum principle, $\hat{a}(u)=w>0$ in $\Omega$ and

$$
\frac{\partial \hat{a}(u)}{\partial n}=a(u) \frac{\partial u}{\partial n}<0 \quad \text { in } \partial \Omega
$$

Consequently, $u \in \dot{P}$. To prove the lemma, we argue by contradiction. If $\bar{u}-u \in \partial P$, then, in particular, $\bar{u} \geqslant u$, for every $x \in \bar{\Omega}$ and, since

$$
-\operatorname{div}(A(x) \nabla \hat{a}(\bar{u})) \geqslant f(\lambda, x, \bar{u})
$$


and

$$
-\operatorname{div}(A(x) \nabla \hat{a}(u))=f(\lambda, x, u),
$$

we deduce from the monotonicity of $f$ that

$$
-\operatorname{div}(A(x) \nabla[\hat{a}(\bar{u})-\hat{a}(u)]) \geqslant f(\lambda, x, \bar{u})-f(\lambda, x, u) \geqslant 0 .
$$

Hence the strong maximum principle implies that

$$
\hat{a}(\bar{u})-\hat{a}(u)>0 \quad \text { in } \Omega
$$

and

$$
\frac{\partial(\hat{a}(\bar{u})-\hat{a}(u))}{\partial n}=a(\bar{u}) \frac{\partial \bar{u}}{\partial n}-a(u) \frac{\partial u}{\partial n}<0 \quad \text { in } \partial \Omega .
$$

Moreover, since $\hat{a}$ is strictly increasing, we obtain $\bar{u}>u$ in $\Omega$. By the fact that $a$ is non-increasing and that $u \in \dot{P}$, we deduce that $\partial(\bar{u}-u) / \partial n<0$. Then $\bar{u}-u \in \dot{P}$, which is a contradiction, proving the lemma and thus the theorem.

We can now give a partial positive answer to the above question with the following result.

TheOREM 5.16. Suppose, in addition to the hypotheses of theorem 5.12, condition $\left(A_{6}\right)$ holds and that $f$ is non-decreasing in $\lambda$ and $s$. If there exists a positive non-decreasing function $\ell$ satisfying

$$
f(\lambda, x, s)<0 \quad \forall \lambda<-\ell(s) \quad \text { a.e. } x \in \Omega, s \in \mathbb{R}^{+},
$$

then the constants $\Lambda^{*}$ and $\bar{\lambda}$ given in theorem 5.12 are equal, i.e. problem $\left(P_{\lambda}\right)$ has positive solutions if and only if $\lambda \leqslant \bar{\lambda}=\Lambda^{*}$.

Proof. We claim that if $u_{n}$ is a sequence of positive solutions of $\left(P_{\lambda}\right)$ for $\lambda=\Lambda$ with $\lambda_{n} \rightarrow-\infty$, then $\left\|u_{n}\right\| \rightarrow+\infty$. Indeed, if, by contradiction, $\left\|u_{n}\right\|_{0} \leqslant s_{0}$, then, for $\lambda_{n}<-\ell\left(s_{0}\right)$, we would have $f\left(\lambda_{n}, x, u_{n}\right)<0$, and taking $u_{n}$ as a test function, by $\left(A_{2}\right)$, we would have that $u_{n}=0$, a contradiction proving the claim. Thus the unbounded continuum $\Sigma_{0}$ of positive solutions emanating from zero connects $\left(\lambda_{0}, 0\right)$ and $(-\infty,+\infty)$. In order to prove the theorem, it suffices to show that if, for some $\Lambda>\lambda_{0}$, problem $\left(P_{\lambda}\right)$ for $\lambda=\Lambda$ admits a positive solution, then $\Lambda \in \operatorname{Proj} \Sigma_{0}$. Suppose that $\left(P_{\lambda}\right)$ for $\lambda=\Lambda$ has a positive solution $u_{\Lambda}$. Then $u_{\Lambda}$ is supersolution of $\left(P_{\lambda}\right)$ for all $\lambda \in(-\infty, \Lambda) \equiv I$ and, taking $T(\lambda)=u_{\Lambda}$ for every $\lambda<\Lambda$, we deduce from theorem 5.14 that $\Sigma_{0} \cap(I \times E)$ does not cross $u=u_{\Lambda}$ and the only way to connect $\left(\lambda_{0}, 0\right)$ and $(-\infty,+\infty)$ is that $\Sigma_{0}$ cross the region $\lambda \geqslant \Lambda$. In particular, $\Lambda \in \operatorname{Proj} \Sigma_{0}$.

\section{Acknowledgments}

This work has been developed while the J.C. and B.P. were visiting the Departamento de Análisis Matemático, Universidad de Granada. They gratefully acknowledge the whole department for the warm hospitality and the friendly atmosphere.

D.A. was partly supported by EEC contract no. ERBCHRXCT940494 and by Acción Integrada Spain-Italy HI1997-0049 . D.A. and J.C. were partly supported 
by DGES Ministerio de Educación y Ciencia (Spain) No. PB98-1283. B.P. was also partly supported by Cofin. National Project 'Variational methods and nonlinear differential equations' and Acción Integrada Spain-Italy HI1997-0049.

\section{References}

1 A. Ambrosetti and P. Hess. Positive solutions of asymptotically linear elliptic eigenvalue problems. J. Math. Analysis Appl. 73 (1980), 411-422.

A. Ambrosetti, H. Brézis and G. Cerami. Combined effects of concave and convex nonlinearities in some elliptic problems. J. Funct. Analysis 122 (1994), 519-543.

A. Ambrosetti, J. G. Azorero and I. Peral. Multiplicity results for some nonlinear elliptic equations. J. Funct. Analysis 137 (1996), 219-242.

4 A. Ambrosetti, J. G. Azorero and I. Peral. Quasi-linear equations with a multiple bifurcation. Diff. Integ. Eqns 10 (1997), 37-50.

5 D. Arcoya and J. L. Gámez. Bifurcation theory and related problems: anti-maximum principle and resonance (1998). (Preprint.)

6 M. Artola. Sur une classe de problémes paraboliques quasi-linéares. Boll. UMI B 5 (1986), 51-70.

7 M. Artola and L. Boccardo. Positive solutions for some quasi-linear elliptic equations. Commun. Appl. Nonlinear Analysis 3(4), (1996), 89-98.

8 L. Boccardo. Positive eigenfunctions for a class of quasi-linear operators. Boll. UMI B 5 (1981), 951-959.

9 H. Brézis and S. Kamin. Sublinear elliptic equations in $\mathbb{R}^{N}$. Manuscr. Math. 74 (1992), $87-106$.

10 H. Brézis and L. Oswald. Remarks on sublinear elliptic equations. Nonlinear Analysis 10 (1986), 55-64.

11 S. Campanato. Equazioni ellittiche del secondo ordine e spazi $\mathcal{L}^{2, \lambda}$. Ann. Mat. 69 (1965), $321-382$.

D. G. De Figueiredo. Positive solutions of semilinear elliptic problems. In Proc. 1st Latin American School of Differential Equations. Lecture Notes 957, 1.982.

13 E. De Giorgi. Sulla differenziabilitáa e l'analiticitá degli estremali degli integrali multipli regolari. Mem. Acad. Sci. Torino (1957), 25-43.

14 P. Drábek and S. I. Pohozaev. Positive solutions for the $p$-Laplacian: application of the fibering method. Proc. R. Soc. Edinb. A 127 (1997), 703-726.

15 J. L. Gámez. Sub- and super-solutions in bifurcation problems. Nonlinear Analysis 28 (1997), 625-632.

16 B. Gidas and J. Spruck. A priori bounds for positive solutions of nonlinear elliptic equations. Commun. PDEs 6 (1981), 883-901.

D. Gilbarg and N. Trudinger. Elliptic partial differential equations of second order (Springer, 1983).

E. Giusti. Equazioni ellittiche del secondo ordine. Quad. Unione Mat. Ital. 6 (1978).

J. Leray and J. L. Lions. Quelques résultats de Višik sur le problemes elliptiques non linéaires par les méthodes de Minty-Browder. Bull. Soc. Math. France 93 (1965), 97-107. P. H. Rabinowitz. Some global results for nonlinear eigenvalue problems. J. Funct. Analysis 7 (1971), 487-513.

G. Stampacchia. Le probléme de Dirichlet pour les équations elliptiques du second ordre á coefficients discontinus. Ann. Inst. Fourier Grenoble 15 (1965), 189-258. 